\title{
INFINITE SERIES ASYMPTOTIC EXPANSIONS FOR DECAYING SOLUTIONS OF DISSIPATIVE DIFFERENTIAL EQUATIONS WITH NON-SMOOTH NONLINEARITY
}

\author{
DAT $\mathrm{CAO}^{1}$, LUAN HOANG ${ }^{2, *}$, AND THINH KIEU ${ }^{3}$
}

\begin{abstract}
We study the precise asymptotic behavior of a non-trivial solution that converges to zero, as time tends to infinity, of dissipative systems of nonlinear ordinary differential equations. The nonlinear term of the equations may not possess a Taylor series expansion about the origin. This absence technically cripples previous proofs in establishing an asymptotic expansion, as an infinite series, for such a decaying solution. In the current paper, we overcome this limitation and obtain an infinite series asymptotic expansion, as time goes to infinity. This series expansion provides large time approximations for the solution with the errors decaying exponentially at any given rates. The main idea is to shift the center of the Taylor expansions for the nonlinear term to a non-zero point. Such a point turns out to come from the non-trivial asymptotic behavior of the solution, which we prove by a new and simple method. Our result applies to different classes of non-linear equations that have not been dealt with previously.
\end{abstract}

\section{Contents}

1. Introduction

2. Notation, definitions and background

3. The first asymptotic approximation

4. The series expansion

5. Extended results

6. Specific cases and examples

References

\section{INTRODUCTION}

The Navier-Stokes equations (NSE) for a viscous, incompressible fluid in bounded or periodic domains with a potential body force can be written in the functional form as

$$
\frac{\mathrm{d} y}{\mathrm{~d} t}+A y+B(y, y)=0,
$$

where $A$ is the (linear) Stokes operator and $B$ is a bilinear form in appropriate functional spaces.

In [18], Foias and Saut prove that any regular solution $y(t)$ of (1.1) has a following asymptotic behavior, as $t \rightarrow \infty$,

$$
e^{\lambda t} y(t) \rightarrow \xi \text { for some } \lambda>0 \text { and } \xi \neq 0 \text { with } A \xi=\lambda \xi .
$$

Date: July 5, 2021.

${ }^{*}$ Corresponding author. 
This result is extended later by Ghidaglia [21] to a more general class of parabolic inequalities. The proof in [21] uses the same Dirichlet quotient technique by Foias-Saut [18].

In [19], Foias and Saut go further and prove the following asymptotic expansion, as $t \rightarrow \infty$,

$$
y(t) \sim \sum_{n=1}^{\infty} q_{n}(t) e^{-\mu_{n} t},
$$

in all Sobolev spaces, where $q_{n}(t)$ are polynomials in $t$, valued in the space of smooth functions. See Definition 2.1 below for the precise meaning of (1.3). Their proof of (1.3) does not require the knowledge of (1.2) and uses a completely different technique.

The expansion (1.3) is studied deeply in later work [15 17,20] concerning its convergence, associated normalization map, normal form, invariant nonlinear manifolds, relation with the Poincaré-Dulac theory, etc. It is applied to the analysis of physics-oriented aspects of fluid flows [13, 14, is established for the NSE in different contexts such as with the Coriolis force [25], or with non-potential forces [8, 10, 24], is extended to dissipative wave equations in [27], is investigated for general ordinary differential equations (ODE) without forcing functions in [26], and with forcing functions in [9]. The considerations of ODE in [9, 26] turns out to be fruitful, and prompts to the recently obtained asymptotic expansions for the Lagrangian trajectories of viscous, incompressible fluid flows in [23].

In the same spirit as [9, 26], we study, in this paper, the ODE systems in $\mathbb{R}^{d}$ of the form

$$
\frac{\mathrm{d} y}{\mathrm{~d} t}+A y=F(y), \quad t>0
$$

where $A$ is a $d \times d$ constant (real) matrix, and $F$ is a vector field on $\mathbb{R}^{d}$.

Our goal is to obtain the asymptotic expansion (1.3), as $t \rightarrow \infty$, for any decaying solution $y(t)$ of (1.4), where $q_{n}(t)$ 's are $\mathbb{R}^{d}$-valued polynomials in $t$. (For other approaches to the asymptotic analysis of the solutions, see discussions in Remark 6.14 below.)

In all of the above cited papers, function $F$ in (1.4) must be infinitely differentiable at the origin. It is due to the requirement that $F(y)$ can be approximated, up to arbitrary orders, near the limit of $y(t)$, i.e. the origin, by the polynomials that come from of the Taylor series of $F$. The current paper investigates the situation when this is not the case, and hence the results in [9, 26, 27] do not apply.

A standard and intuitive way to find expansion (1.3) is substituting it into equation (1.4), expanding both sides in $t$, and equating the coefficient functions of corresponding exponential terms. Because of the lack of the Taylor series of $F(y)$ about the origin, one does not know how to find the expansion in $t$ for $F(y(t))$ on the right-hand side of (1.5). The task seems to be impossible. However, as will be proved later in this paper, we are still able to obtain the infinite series asymptotic expansion (1.3) for $y(t)$ in many cases. This is achieved by combining Foias-Saut's method in [19] with the following new idea. For illustrative purposes, we consider an example,

$$
\frac{\mathrm{d} y}{\mathrm{~d} t}+A y=F(y)=\frac{|y|^{1 / 3} y}{1+|y|^{1 / 4}}
$$

First, we use the geometric series to approximate $F(y)$ by a series

$$
F(y) \sim \sum_{k=1}^{\infty} F_{k}(y) \text { as } y \rightarrow 0
$$


where $F_{k}$ 's are a positively homogeneous functions of strictly increasing degrees $\beta_{k} \rightarrow \infty$. (In general cases, (1.6) is a hypothesis.) See Definition 2.2 and Assumption 4.1 for details. After establishing the asymptotic approximation (1.2) for some eigenvector $\xi$ of $A$, we approximate each $F_{k}$ by using its Taylor series about $\xi \neq 0$. Therefore, we can bypass the lack of the Taylor series of $F$ about 0 . This, of course, is just a brief description and must be facilitated with capable techniques.

The paper is organized as follows. In section 2, we set the assumptions for matrix $A$, establish basic properties and recall a crucial approximation lemma, Lemma 2.4.

In section 3, we prove, for a more general equation (3.1) with a general structure (3.2), that any non-trivial, decaying solution has the first asymptotic approximation (1.2), see Theorem 3.3. This result can be obtained by repeating Foias-Saut's proof in [18, Proposition 3], or applying [21, Theorem 1.1]. However, our new proof provides an alternative method and, at least for the current setting, is shorter. See Remark 3.4 for comparisons between the proofs.

The paper's main result is in section 4. In Theorem 4.3, we prove that any non-trivial, decaying solution of (1.4) has an asymptotic expansion of the form (1.3). In order to implement to general scheme of Foias-Saut's [19], we use the first approximation $e^{-\lambda t} \xi$ in (1.2). By the positive homogeneity of each function $F_{k}$ in (1.6), we can scale $y(t)$ by the factor $e^{-\lambda t}$ and then shift the Taylor expansions of $F_{k}$ 's from center zero to center $\xi \neq 0$. Because of the above scaling and its effect during complicated iterations, the exponential rates must be

shifted back, see the set $\widetilde{S}$ in (4.8), and forth, see the set $S$ in (4.10), when being generated in Definition 4.2 .

Although we focus on infinite series expansions in this paper, we consider, in the first part of section 5, the case when the function $F(y)$ has only a finite sum approximation, see (5.1). We prove in Theorem 5.1 that any decaying solution $y(t)$ has a corresponding finite sum approximation. In the second part of section 5 , Theorem 5.3 generalizes Theorems 4.3 and 5.1 by relaxing the conditions on functions $F$ and $F_{k}$ 's, in accordance with the knowledge of the eigenspaces of $A$.

Section 6 is devoted to identifying some specific classes of functions $F$, see Theorems 6.1, 6.5 and 6.9. Briefly speaking, these functions can be expanded in terms of power-like functions of the types $x_{i}^{\gamma_{i}},\left|x_{i}\right|^{\gamma_{i}},\left|x_{i}\right|^{\gamma_{i}} \operatorname{sign}\left(x_{i}\right)$ for coordinates $x_{i}$ 's of $x \in \mathbb{R}^{d}$, or of type $\|x\|_{p}^{\gamma}$, or, more generally, $\|P(x)\|_{p}^{\gamma}$ with $\ell^{p}$-norms $\|\cdot\|_{p}$, where $P$ is a homogeneous polynomial. Lastly, we compare, in Remark 6.14, our results with other asymptotic expansion theories for ODE, notably the one that has been developed by Bruno and collaborators, see [3, 4, 6, ,7] and references therein.

\section{Notation, Definitions AND BACKGRound}

We will use the following notation throughout the paper.

- $\mathbb{N}=\{1,2,3, \ldots\}$ denotes the set of natural numbers, and $\mathbb{Z}_{+}=\mathbb{N} \cup\{0\}$.

- Denote $\mathbb{R}_{*}=\mathbb{R} \backslash\{0\}$, and, for $n \in \mathbb{N}, \mathbb{R}_{*}^{n}=\left(\mathbb{R}_{*}\right)^{n}$ and $\mathbb{R}_{0}^{n}=\mathbb{R}^{n} \backslash\{0\}$.

- For any vector $x \in \mathbb{R}^{n}$, we denote by $|x|$ its Euclidean norm, and by $x^{(k)}$ the $k$-tuple $(x, \ldots, x)$ for $k \geq 1$, and $x^{(0)}=1$.

- For an $m \times n$ matrix $M$, its Euclidean norm in $\mathbb{R}^{m n}$ is denoted by $|M|$.

- Let $f$ be an $\mathbb{R}^{m}$-valued function and $h$ be a non-negative function, both are defined in a neighborhood of the origin in $\mathbb{R}^{n}$. We write $f(x)=\mathcal{O}(h(x))$ as $x \rightarrow 0$, if there are positive numbers $r$ and $C$ such that $|f(x)| \leq C h(x)$ for all $x \in \mathbb{R}^{n}$ with $|x|<r$. 
- Let $f:\left[T_{0}, \infty\right) \rightarrow \mathbb{R}^{n}$ and $h:\left[T_{0}, \infty\right) \rightarrow[0, \infty)$ for some $T_{0} \in \mathbb{R}$. We write $f(t)=\mathcal{O}(h(t))$, implicitly meaning as $t \rightarrow \infty$,

if there exist numbers $T \geq T_{0}$ and $C>0$ such that $|f(t)| \leq C h(t)$ for all $t \geq T$.

- Let $T_{0} \in \mathbb{R}$, functions $f, g:\left[T_{0}, \infty\right) \rightarrow \mathbb{R}^{n}$, and $h:\left[T_{0}, \infty\right) \rightarrow[0, \infty)$. We will conveniently write $f(t)=g(t)+\mathcal{O}(h(t))$ to indicate $f(t)-g(t)=\mathcal{O}(h(t))$.

The type of asymptotic expansions at time infinity that is studied in this paper is the following.

Definition 2.1. Let $\left(X,\|\cdot\|_{X}\right)$ be a normed space and $\left(\alpha_{n}\right)_{n=1}^{\infty}$ be a sequence of strictly increasing non-negative real numbers. A function $f:[T, \infty) \rightarrow X$, for some $T \geq 0$, is said to have an asymptotic expansion

$$
f(t) \sim \sum_{n=1}^{\infty} f_{n}(t) e^{-\alpha_{n} t} \quad \text { in } X,
$$

where each $f_{n}: \mathbb{R} \rightarrow X$ is a polynomial, if one has, for any $N \geq 1$, that

$$
\left\|f(t)-\sum_{n=1}^{N} f_{n}(t) e^{-\alpha_{n} t}\right\|_{X}=\mathcal{O}\left(e^{-\left(\alpha_{N}+\varepsilon_{N}\right) t}\right) \text { for some } \varepsilon_{N}>0 .
$$

One can see, e.g. [9, Lemma 4.1], that the polynomials $f_{1}, f_{2}, \ldots, f_{N}$ in (2.2) are unique.

In the case $\alpha_{n} \rightarrow \infty$ as $n \rightarrow \infty$, the (infinite series) asymptotic expansion (2.1) provides exponentially precise approximations for $f(t)$, as $t \rightarrow \infty$. More specifically, for any $\gamma>0$, the partial sum $\sum_{n=1}^{N} f_{n}(t) e^{-\alpha_{n} t}$ of the series, with sufficiently large $N$, approximates $f(t)$, as $t \rightarrow \infty$, with an error of order $\mathcal{O}\left(e^{-\gamma t}\right)$.

Regarding the nonlinearity in (1.4), the function $F$ will be approximated near the origin by functions, not necessarily polynomials, in the following class.

Definition 2.2. Suppose $\left(X,\|\cdot\|_{X}\right)$ and $\left(Y,\|\cdot\|_{Y}\right)$ be two (real) normed spaces.

A function $F: X \rightarrow Y$ is positively homogeneous of degree $\beta \geq 0$ if

$$
F(t x)=t^{\beta} F(x) \text { for any } x \in X \text { and any } t>0 \text {. }
$$

Define $\mathcal{H}_{\beta}(X, Y)$ to be the set of positively homogeneous functions of order $\beta$ from $X$ to $Y$, and denote $\mathcal{H}_{\beta}(X)=\mathcal{H}_{\beta}(X, X)$.

For a function $F \in \mathcal{H}_{\beta}(X, Y)$, define

$$
\|F\|_{\mathcal{H}_{\beta}}=\sup _{\|x\|_{X}=1}\|F(x)\|_{Y}=\sup _{x \neq 0} \frac{\|F(x)\|_{Y}}{\|x\|_{X}^{\beta}} .
$$

The following are immediate properties.

(a) If $F \in \mathcal{H}_{\beta}(X, Y)$ with $\beta>0$, then taking $x=0$ and $t=2$ in (2.3) gives

$$
F(0)=0 \text {. }
$$

If, in addition, $F$ is bounded on the unit sphere in $X$, then

$$
\|F\|_{\mathcal{H}_{\beta}} \in[0, \infty) \text { and }\|F(x)\|_{Y} \leq\|F\|_{\mathcal{H}_{\beta}}\|x\|_{X}^{\beta} \quad \forall x \in X .
$$

(b) The zero function (from $X$ to $Y$ ) belongs to $\mathcal{H}_{\beta}(X, Y)$ for all $\beta \geq 0$, and a constant function (from $X$ to $Y$ ) belongs to $\mathcal{H}_{0}(X, Y)$.

(c) Each $\mathcal{H}_{\beta}(X, Y)$, for $\beta \geq 0$, is a linear space.

(d) If $F_{1} \in \mathcal{H}_{\beta_{1}}(X, \mathbb{R})$ and $F_{2} \in \mathcal{H}_{\beta_{2}}(X, Y)$, then $F_{1} F_{2} \in \mathcal{H}_{\beta_{1}+\beta_{2}}(X, Y)$. 
(e) If $F: X \rightarrow Y$ is a homogeneous polynomial of degree $m \in \mathbb{Z}_{+}$, then $F \in \mathcal{H}_{m}(X, Y)$. In (e) above and throughout the paper, a constant function, even when it is zero, is considered as a homogeneous polynomial of degree zero.

The space $\mathcal{H}_{\beta}(X, Y)$ can contain much more complicated functions than homogeneous polynomials. For example, let $s \in \mathbb{Z}_{+}$, numbers $\nu_{j}$, for $1 \leq j \leq s$, be positive, $P_{j}$, for $1 \leq j \leq s$, be a homogeneous polynomial of degree $m_{j} \in \mathbb{N}$ from $X$ to a normed space $\left(Y_{j},\|\cdot\|_{Y_{j}}\right)$. Let $P_{0}: X \rightarrow Y$ be homogeneous polynomial of degree $m_{0} \in \mathbb{Z}_{+}$. Consider function $F$ defined by

$$
F(x)=\left\|P_{1}(x)\right\|_{Y_{1}}^{\nu_{1}}\left\|P_{2}(x)\right\|_{Y_{2}}^{\nu_{2}} \ldots\left\|P_{s}(x)\right\|_{Y_{s}}^{\nu_{s}} P_{0}(x), \text { for } x \in X .
$$

Then one has

$$
F \in \mathcal{H}_{\beta}(X, Y), \text { where } \beta=m_{0}+\sum_{j=1}^{s} m_{j} \nu_{j}
$$

Thanks to (2.7) and property (c) above, any linear combination of functions of the form in (2.6) with the same number $\beta$ also belongs to $\mathcal{H}_{\beta}(X, Y)$. by

If $n, m, k \in \mathbb{N}$ and $\mathcal{L}$ is an $m$-linear mapping from $\left(\mathbb{R}^{n}\right)^{m}$ to $\mathbb{R}^{k}$, the norm of $\mathcal{L}$ is defined

$$
\|\mathcal{L}\|=\max \left\{\left|\mathcal{L}\left(x_{1}, x_{2}, \ldots, x_{m}\right)\right|: x_{j} \in \mathbb{R}^{n},\left|x_{j}\right|=1, \text { for } 1 \leq j \leq m\right\} .
$$

It is known that the norm $\|\mathcal{L}\|$ belongs to $[0, \infty)$, and one has

$$
\left|\mathcal{L}\left(x_{1}, x_{2}, \ldots, x_{m}\right)\right| \leq\|\mathcal{L}\| \cdot\left|x_{1}\right| \cdot\left|x_{2}\right| \ldots\left|x_{m}\right| \quad \forall x_{1}, x_{2}, \ldots, x_{m} \in \mathbb{R}^{n} .
$$

In particular, when $m=1$, (2.8) yields the operator norm for any $k \times n$ matrix $\mathcal{L}$.

Let the space's dimension $d \in \mathbb{N}$ be fixed throughout the paper. Consider the ODE system (1.4).

Assumption 2.3. Hereafter, matrix $A$ is a diagonalizable with positive eigenvalues.

Thanks to Assumption 2.3, the spectrum $\sigma(A)$ of matrix $A$ consists of eigenvalues $\Lambda_{k}$ 's, for $1 \leq k \leq d$, which are positive and increasing in $k$. Then there exists an invertible matrix $S$ such that

$$
A=S^{-1} A_{0} S, \text { where } A_{0}=\operatorname{diag}\left[\Lambda_{1}, \Lambda_{2}, \ldots, \Lambda_{d}\right] .
$$

Denote the distinct eigenvalues of $A$ by $\lambda_{j}$ 's that are strictly increasing in $j$, i.e.,

$$
0<\lambda_{1}=\Lambda_{1}<\lambda_{2}<\ldots<\lambda_{d_{*}}=\Lambda_{d} \quad \text { with } 1 \leq d_{*} \leq d .
$$

For $1 \leq k, \ell \leq d$, let $E_{k \ell}$ be the elementary $d \times d$ matrix $\left(\delta_{k i} \delta_{\ell j}\right)_{1 \leq i, j \leq d}$, where $\delta_{k i}$ and $\delta_{\ell j}$ are the Kronecker delta symbols.

For $\lambda \in \sigma(A)$, define

$$
\hat{R}_{\lambda}=\sum_{1 \leq i \leq d, \Lambda_{i}=\lambda} E_{i i} \text { and } R_{\lambda}=S^{-1} \hat{R}_{\lambda} S .
$$

Then one immediately has

$$
I_{d}=\sum_{j=1}^{d_{*}} R_{\lambda_{j}}, \quad R_{\lambda_{i}} R_{\lambda_{j}}=\delta_{i j} R_{\lambda_{j}}, \quad A R_{\lambda_{j}}=R_{\lambda_{j}} A=\lambda_{j} R_{\lambda_{j}}
$$


and there exists $c_{0} \geq 1$ such that

$$
c_{0}^{-1}|x|^{2} \leq \sum_{j=1}^{d_{*}}\left|R_{\lambda_{j}} x\right|^{2} \leq c_{0}|x|^{2} \text { for all } x \in \mathbb{R}^{d} .
$$

Below, we recall a key approximation lemma for linear ODEs. It is Lemma 2.2 of [9], which originates from Foias-Saut's work [19], and is based on the first formalized version [24, Lemma 4.2].

Lemma 2.4 ( [9, Lemma 2.2]). Let $p(t)$ be an $\mathbb{R}^{d}$-valued polynomial and $g:[T, \infty) \rightarrow \mathbb{R}^{d}$, for some $T \in \mathbb{R}$, be a continuous function satisfying $|g(t)|=\mathcal{O}\left(e^{-\alpha t}\right)$ for some $\alpha>0$. Suppose $\lambda>0$ and $y \in C\left([T, \infty), \mathbb{R}^{d}\right)$ is a solution of

$$
y^{\prime}(t)=-\left(A-\lambda I_{d}\right) y(t)+p(t)+g(t), \quad \text { for } t \in(T, \infty) .
$$

If $\lambda>\lambda_{1}$, assume further that

$$
\lim _{t \rightarrow \infty}\left(e^{(\bar{\lambda}-\lambda) t}|y(t)|\right)=0 \text {, where } \bar{\lambda}=\max \left\{\lambda_{j}: 1 \leq j \leq d_{*}, \lambda_{j}<\lambda\right\} .
$$

Then there exists a unique $\mathbb{R}^{d}$-valued polynomial $q(t)$ such that

$$
q^{\prime}(t)=-\left(A-\lambda I_{d}\right) q(t)+p(t) \text { for } t \in \mathbb{R},
$$

and

$$
|y(t)-q(t)|=\mathcal{O}\left(e^{-\varepsilon t}\right) \text { for some } \varepsilon>0 .
$$

In fact, the polynomial $q(t)$ in Lemma 2.4 can be defined explicitly as follows. We write, with the use of (2.10), $q(t)=\sum_{j=1}^{d_{*}} R_{\lambda_{j}} q(t)$, where, for each $1 \leq j \leq d_{*}$ and $t \in \mathbb{R}$,

$$
R_{\lambda_{j}} q(T+t)= \begin{cases}e^{-\left(\lambda_{j}-\lambda\right) t} \int_{0}^{t} e^{\left(\lambda_{j}-\lambda\right) \tau} R_{\lambda_{j}} p(T+\tau) d \tau & \text { if } \lambda_{j}>\lambda, \\ R_{\lambda_{j}} y(T)+\int_{0}^{\infty} R_{\lambda_{j}} g(T+\tau) d \tau+\int_{0}^{t} R_{\lambda_{j}} p(T+\tau) d \tau & \text { if } \lambda_{j}=\lambda, \\ -e^{-\left(\lambda_{j}-\lambda\right) t} \int_{t}^{\infty} e^{\left(\lambda_{j}-\lambda\right) \tau} R_{\lambda_{j}} p(T+\tau) d \tau & \text { if } \lambda_{j}<\lambda .\end{cases}
$$

In the case $p(t) \equiv 0$, it follows (2.15) that $q(t) \equiv \xi$, which is a constant vector in $\mathbb{R}^{d}$. Then (2.13) and (2.14) read as

$$
\left(A-\lambda I_{d}\right) \xi=0 \text { and }|y(t)-\xi|=\mathcal{O}\left(e^{-\varepsilon t}\right) .
$$

\section{THE FIRST ASYMPTOTIC APPROXIMATION}

Consider the following ODE on $\mathbb{R}^{d}$, which is more general than (1.4),

$$
\frac{\mathrm{d} y}{\mathrm{~d} t}+A y=F(t, y), \quad t>0 .
$$

Assumption 3.1. Function $F$ mapping $(t, x) \in[0, \infty) \times \mathbb{R}^{d}$ to $F(t, x) \in \mathbb{R}^{d}$ is continuous in $[0, \infty) \times \mathbb{R}^{d}$, locally Lipschitz with respect to $x$ in $[0, \infty) \times \mathbb{R}^{d}$, and there exist positive numbers $c_{*}, \varepsilon_{*}$, $\alpha$ such that

$$
|F(t, x)| \leq c_{*}|x|^{1+\alpha} \forall t \geq 0, \forall x \in \mathbb{R}^{d} \text { with }|x| \leq \varepsilon_{*}
$$

It follows (3.2) that $F(t, 0)=0$ for all $t \geq 0$. By the uniqueness/backward uniqueness of ODE system (3.1), a solution $y(t) \in C^{1}([0, \infty))$ of (3.1) has the property

$$
y(0)=0 \text { if and only if } y(t)=0 \text { for all } t \geq 0 \text {. }
$$


Thanks to Assumption 2.3 and (3.2), it is well-known that the trivial solution $y(t) \equiv 0$ of (1.4) is asymptotically stable, see, for example, [11, Theorem 1.1, Chapter 13].

A solution $y(t) \in C^{1}([0, \infty))$ of (3.1) that satisfies $y(0) \neq 0$ and

$$
\lim _{t \rightarrow \infty} y(t)=0
$$

will be referred to as a non-trivial, decaying solution. These solutions will be the focus of our study.

The following elementary result provides, for non-trivial, decaying solutions, a more precise upper bound, compared to (3.4), and an additional lower bound.

Proposition 3.2. Let $y(t)$ be a non-trivial, decaying solution of (3.1). Then there exists a number $C_{1}>0$ such that

$$
|y(t)| \leq C_{1} e^{-\Lambda_{1} t} \text { for all } t \geq 0 .
$$

Moreover, for any $\varepsilon>0$, there exists a number $C_{2}=C_{2}(\varepsilon)>0$ such that

$$
|y(t)| \geq C_{2} e^{-\left(\Lambda_{d}+\varepsilon\right) t} \text { for all } t \geq 0
$$

Proof. Set $Y(t)=\left(\sum_{j=1}^{d_{*}}\left|R_{\lambda_{j}} y(t)\right|^{2}\right)^{1 / 2}$. Applying $R_{\lambda_{j}}$ to equation (3.1), taking dot product of the resulting equation with $R_{\lambda_{j}} y$, using the last property in (2.10), and then summing over $j$, we obtain

$$
\frac{1}{2} \frac{\mathrm{d}}{\mathrm{d} t} Y^{2}(t)=\frac{1}{2} \frac{\mathrm{d}}{\mathrm{d} t} \sum_{j=1}^{d_{*}}\left|R_{\lambda_{j}} y\right|^{2}=-\sum_{j=1}^{d_{*}} \lambda_{j}\left|R_{\lambda_{j}} y\right|^{2}+\sum_{j=1}^{d_{*}} R_{\lambda_{j}} F(t, y) \cdot R_{\lambda_{j}} y .
$$

Note that

$$
\Lambda_{1} \sum_{j=1}^{d_{*}}\left|R_{\lambda_{j}} y\right|^{2} \leq \sum_{j=1}^{d_{*}} \lambda_{j}\left|R_{\lambda_{j}} y\right|^{2} \leq \Lambda_{d} \sum_{j=1}^{d_{*}}\left|R_{\lambda_{j}} y\right|^{2} .
$$

Denote $C_{0}=\sum_{j=1}^{d_{*}}\left\|R_{\lambda_{j}}\right\|^{2}$. Let $\varepsilon>0$ be arbitrary. By (3.4) and the asymptotic stability of the trivial solution, there exists $T_{\varepsilon} \geq 0$ such that

$$
|y(t)| \leq \varepsilon_{*} \text { and } C_{0} c_{*} c_{0}|y(t)|^{\alpha} \leq \varepsilon \forall t \geq T_{\varepsilon} .
$$

We have, for $t \geq T_{\varepsilon}$,

$$
\left|\sum_{j=1}^{d_{*}} R_{\lambda_{j}} F(t, y) \cdot R_{\lambda_{j}} y\right| \leq \sum_{j=1}^{d_{*}}\left\|R_{\lambda_{j}}\right\|^{2}|F(t, y)| \cdot|y| \leq C_{0} c_{*}|y|^{2+\alpha} .
$$

Combining (3.10) with (2.11) and (3.9) gives

$$
\left|\sum_{j=1}^{d_{*}} R_{\lambda_{j}} F(t, y) \cdot R_{\lambda_{j}} y\right| \leq C_{0} c_{*}|y|^{\alpha} \cdot c_{0} Y^{2}(t) \leq \varepsilon Y^{2}(t) \forall t \geq T_{\varepsilon} .
$$

Proof of (3.5). By equation (3.7), the first inequality in (3.8), and (3.11), we have

$$
\frac{1}{2} \frac{\mathrm{d}}{\mathrm{d} t} Y^{2} \leq-\left(\Lambda_{1}-\varepsilon\right) Y^{2} \forall t \geq T_{\varepsilon} .
$$

Thus, for $t \geq T_{\varepsilon}$,

$$
Y^{2}(t) \leq Y^{2}\left(T_{\varepsilon}\right) e^{-2\left(\Lambda_{1}-\varepsilon\right)\left(t-T_{\varepsilon}\right)}
$$


Using this estimate and (3.10) in (3.7) gives, for $t>T_{\varepsilon}$,

$$
\frac{1}{2} \frac{\mathrm{d}}{\mathrm{d} t} Y^{2} \leq-\Lambda_{1} Y^{2}+C_{0} c_{*}\left(c_{0}^{1 / 2} Y\right)^{2+\alpha} \leq-\Lambda_{1} Y^{2}+C_{1}^{\prime} e^{-(2+\alpha)\left(\Lambda_{1}-\varepsilon\right)\left(t-T_{\varepsilon}\right)},
$$

hence,

$$
\frac{\mathrm{d}}{\mathrm{d} t} Y^{2} \leq-2 \Lambda_{1} Y^{2}+2 C_{1}^{\prime} e^{-2 \beta\left(t-T_{\varepsilon}\right)}
$$

where $\beta=(1+\alpha / 2)\left(\Lambda_{1}-\varepsilon\right)$ and $C_{1}^{\prime}$ is a positive number.

Choose $\varepsilon$ sufficiently small so that $\beta>\Lambda_{1}$. Applying Gronwall's inequality to (3.12), for $t \geq T_{\varepsilon}$, yields

$$
Y^{2}(t) \leq e^{-2 \Lambda_{1}\left(t-T_{\varepsilon}\right)} Y^{2}\left(T_{\varepsilon}\right)+2 C_{1}^{\prime} \int_{T_{\varepsilon}}^{t} e^{-2 \Lambda_{1}(t-\tau)} e^{-2 \beta\left(\tau-T_{\varepsilon}\right)} \mathrm{d} \tau,
$$

and, also by (2.11),

$$
|y(t)|^{2} \leq c_{0} Y^{2}(t) \leq e^{-2 \Lambda_{1}\left(t-T_{\varepsilon}\right)} c_{0}\left(Y^{2}\left(T_{\varepsilon}\right)+\frac{C_{1}^{\prime}}{\beta-\Lambda_{1}}\right)
$$

Therefore, we obtain the inequality in (3.5) for some constant $C_{1}>0$, but only for all $t \geq T_{\varepsilon}$. Combining this with the boundedness of $|y(t)|$ on $\left[0, T_{\varepsilon}\right]$, we then obtain estimate (3.5) for all $t \geq 0$ with an adjusted constant $C_{1}>0$.

Proof of (3.6). By equation (3.7), the second inequality in (3.8), and (3.11), we have

$$
\frac{1}{2} \frac{\mathrm{d}}{\mathrm{d} t} Y^{2} \geq-\Lambda_{d} Y^{2}-\varepsilon Y^{2}=-\left(\Lambda_{d}+\varepsilon\right) Y^{2} \quad \forall t>T_{\varepsilon}
$$

Hence,

$$
Y^{2}(t) \geq Y^{2}\left(T_{\varepsilon}\right) e^{-2\left(\Lambda_{d}+\varepsilon\right)\left(t-T_{\varepsilon}\right)} \quad \forall t \geq T_{\varepsilon} .
$$

By the virtue of (3.3) $),|y(t)|>0$ for all $t \geq 0$. It follows that

$$
|y(t)|^{2} \geq c_{0}^{-1} Y^{2}(t) \geq c_{0}^{-2}\left|y\left(T_{\varepsilon}\right)\right|^{2} e^{-2\left(\Lambda_{d}+\varepsilon\right)\left(t-T_{\varepsilon}\right)}=C_{2}^{\prime} e^{-2\left(\Lambda_{d}+\varepsilon\right) t} \quad \forall t \geq T_{\varepsilon},
$$

where $C_{2}^{\prime}>0$. Since $y \in C\left(\left[0, T_{\varepsilon}\right], \mathbb{R}^{d}\right)$ and $|y(t)|>0$ on $\left[0, T_{\varepsilon}\right]$, one has $|y(t)|$ it is bounded below by a positive constant on $\left[0, T_{\varepsilon}\right]$. Combining this fact with estimate (3.13) for $t \geq T_{\varepsilon}$, we obtain the all-time estimate (3.6).

The lower bound (3.6) in Proposition 3.2 can be derived by using results for abstract problems in infinite dimensional spaces such as [22, Theorems 1.1 and 1.2], see also [12]. Nonetheless, the proof above is included for being self-contained and simple.

As discussed in the Introduction, the next theorem either follows the proof of [18, Proposition 3], or is a consequence of [21, Theorem 1.1]. However, the proof presented below uses a new method, which may be useful in other problems.

Theorem 3.3. Let $y(t)$ be a non-trivial, decaying solution of (3.1). Then there exist an eigenvalue $\lambda_{*}$ of $A$ and a corresponding eigenvector $\xi_{*}$ such that

$$
\left|y(t)-e^{-\lambda_{*} t} \xi_{*}\right|=\mathcal{O}\left(e^{-\left(\lambda_{*}+\delta\right) t}\right) \text { for some } \delta>0 .
$$

Proof. Define the set

$$
S^{\prime}=\left\{\sum_{j=1}^{n} \lambda_{j}^{\prime}+m \alpha \lambda_{1}: \text { for any numbers } n \in \mathbb{N}, \lambda_{j}^{\prime} \in \sigma(A), 0 \leq m \in \mathbb{Z}\right\} .
$$


The set $S^{\prime}$ can be arranged as a strictly increasing sequence $\left\{\nu_{n}\right\}_{n=1}^{\infty}$. Note that $\nu_{1}=\lambda_{1}$ and $\nu_{n} \rightarrow \infty$ as $n \rightarrow \infty$. For any $n \in \mathbb{N}$, one has $\nu_{n}+\alpha \lambda_{1}>\nu_{n}$ and $\nu_{n}+\alpha \lambda_{1} \in S^{\prime}$. Hence, by the strict increase of $\nu_{n}$ 's, we have

$$
\nu_{n}+\alpha \lambda_{1} \geq \nu_{n+1} .
$$

Step 1. First, by Proposition [3.2, $|y(t)| \leq C e^{-\nu_{1} t}$. Let $w_{0}(t)=e^{\nu_{1} t} y(t)$. Then $w_{0}(t)$ satisfies

$$
w_{0}^{\prime}(t)+\left(A-\nu_{1} I_{d}\right) w_{0}(t)=g_{1}(t) \stackrel{\text { def }}{=} e^{\nu_{1} t} F(t, y(t)) .
$$

We estimate the right-hand side

$$
\left|g_{1}(t)\right| \leq C e^{\nu_{1} t}|y(t)|^{1+\alpha} \leq C e^{\nu_{1} t} e^{-\nu_{1}(1+\alpha) t}=\mathcal{O}\left(e^{-\alpha \nu_{1} t}\right) .
$$

By equation (3.17) and estimate (3.18), we can apply Lemma 2.4 to $y(t)=w_{0}(t)$ and $p(t) \equiv 0$. Then, by and (2.16), there exists a vector $\xi_{1} \in \mathbb{R}^{d}$ and a number $\varepsilon_{1}>0$ such that

$$
\begin{aligned}
A \xi_{1} & =\nu_{1} \xi_{1}, \\
\left|w_{0}(t)-\xi_{1}\right| & =\mathcal{O}\left(e^{-\varepsilon t}\right), \text { that is },\left|y(t)-e^{-\nu_{1} t} \xi_{1}\right|=\mathcal{O}\left(e^{-\left(\nu_{1}+\varepsilon_{1}\right) t}\right) .
\end{aligned}
$$

Step 2. Set $M=\left\{n \in \mathbb{N}:|y(t)|=\mathcal{O}\left(e^{-\left(\nu_{n}+\delta\right) t}\right)\right.$ for some $\left.\delta>0\right\}$.

Suppose $n \in M$. Let $w_{n}(t)=e^{\nu_{n+1} t} y(t)$. Then

$$
w_{n}^{\prime}(t)+\left(A-\nu_{n+1} I_{d}\right) w_{n}(t)=g_{n+1}(t) \stackrel{\text { def }}{=} e^{\nu_{n+1} t} F(t, y(t)) .
$$

To estimate the last term, we note from (3.16) that $\nu_{n}(1+\alpha) \geq \nu_{n}+\lambda_{1} \alpha \geq \nu_{n+1}$. Then, for large $t$,

$$
\left|g_{n+1}(t)\right| \leq C e^{\nu_{n+1} t}|y(t)|^{1+\alpha} \leq C e^{\nu_{n+1} t} e^{-\left(\nu_{n}+\delta\right)(1+\alpha) t} \mid=\mathcal{O}\left(e^{-\delta(1+\alpha) t}\right) .
$$

By (3.21) and (3.22), we, again, can apply Lemma 2.4 to $y(t)=w_{n}(t)$ and $p(t) \equiv 0$. Then, by (2.16), there exists a vector $\xi_{n+1} \in \mathbb{R}^{d}$ and a number $\varepsilon>0$ such that

$$
\begin{aligned}
A \xi_{n+1} & =\nu_{n+1} \xi_{n+1}, \\
\left|w_{n}(t)-\xi_{n+1}\right| & =\mathcal{O}\left(e^{-\varepsilon t}\right), \text { that is , }\left|y(t)-e^{-\nu_{n+1} t} \xi_{n+1}\right|=\mathcal{O}\left(e^{-\left(\nu_{n+1}+\varepsilon\right) t}\right) .
\end{aligned}
$$

Step 3. If the vector $\xi_{1}$ in Step 1 is not zero, then, thanks to (3.20) and (3.19), the theorem is proved with $\lambda_{*}=\lambda_{1}$ and $\xi_{*}=\xi_{1}$.

Now, consider $\xi_{1}=0$. By (3.20) with $\xi_{1}=0$, one has $1 \in M$, hence $M$ is a non-empty subset of $\mathbb{N}$. By (3.6) and the fact $\nu_{n} \rightarrow \infty$, the set $M$ must be finite. Let $k$ be the maximum number of $M$, and $n_{0}=k+1$. By the result in Step 2 applied to $n=k$, there exist $\xi_{n_{0}} \in \mathbb{R}^{d}$ and $\varepsilon>0$ such that

$$
\begin{gathered}
A \xi_{n_{0}}=\nu_{n_{0}} \xi_{n_{0}}, \\
\left|y(t)-e^{-\nu_{n_{0}} t} \xi_{n_{0}}\right|=\mathcal{O}\left(e^{-\left(\nu_{n_{0}}+\varepsilon\right) t}\right) .
\end{gathered}
$$

If $\xi_{n_{0}}=0$, then (3.24) implies $n_{0} \in M$, which is a contradiction. Thus, $\xi_{n_{0}} \neq 0$, which, together with (3.23), implies $\lambda_{*}=\nu_{n_{0}}$ is an eigenvalue and $\xi_{*}=\xi_{n_{0}}$ is a corresponding eigenvector of $A$. Then, estimate (3.14) follows (3.24).

Remark 3.4. We compare the above proof of Theorem 3.3 with Foias-Saut's proof in [18]. We recall from [18] that the Dirichlet quotient $A y(t) \cdot y(t) /|y(t)|^{2}$ is proved to converge, as $t \rightarrow \infty$, to an eigenvalue $\lambda_{*}$ of $A$ first, and then, based on this, the two limits $e^{\lambda_{*} t} R_{\lambda_{*}} y(t) \rightarrow$ $\xi_{*} \neq 0$ and $e^{\lambda_{*} t}\left(I_{d}-R_{\lambda_{*}}\right) y(t) \rightarrow 0$ are established. This original proof is rather lengthy and requires delicate analysis of the asymptotic behavior of $y(t) /|y(t)|$, see [18, Proposition 1]. 
We, instead, do not use the Dirichlet quotient to determine the exponential rate, but create the set $S^{\prime}$ of possible rates, see (3.15), and find the first $\lambda_{*} \in S^{\prime}$ such that $e^{\lambda_{*} t}|y(t)|$ does not decay exponentially. Then, by the virtue of approximation lemma 2.4, estimate (3.14) is established without analyzing $y(t) /|y(t)|$. This idea, in fact, is inspired by Foias-Saut's proof in [19] of the asymptotic expansion (1.3). However, we restrict it solely to the problem of first asymptotic approximation, and hence make it significantly simpler.

\section{THE SERIES EXPANSION}

In this section, we focus on obtaining the asymptotic expansion, as $t \rightarrow \infty$, for solutions of equation (1.4). Regarding the equation's nonlinearity, we assume the following.

Assumption 4.1. The mapping $F: \mathbb{R}^{d} \rightarrow \mathbb{R}^{d}$ has the the following properties.

(i) $F$ is locally Lipschitz on $\mathbb{R}^{d}$ and $F(0)=0$.

(ii) Either (H1) or (H2) below is satisfied.

(H1) There exist numbers $\beta_{k}$ 's, for $k \in \mathbb{N}$, which belong to $(1, \infty)$ and increase strictly to infinity, and functions $F_{k} \in \mathcal{H}_{\beta_{k}}\left(\mathbb{R}^{d}\right) \cap C^{\infty}\left(\mathbb{R}_{0}^{d}\right)$, for $k \in \mathbb{N}$, such that it holds, for any $N \in \mathbb{N}$, that

$$
\left|F(x)-\sum_{k=1}^{N} F_{k}(x)\right|=\mathcal{O}\left(|x|^{\beta}\right) \text { as } x \rightarrow 0 \text {, for some } \beta>\beta_{N} .
$$

(H2) There exist $N_{*} \in \mathbb{N}$, strictly increasing numbers $\beta_{k}$ 's in $(1, \infty)$, and functions $F_{k} \in \mathcal{H}_{\beta_{k}}\left(\mathbb{R}^{d}\right) \cap C^{\infty}\left(\mathbb{R}_{0}^{d}\right)$, for $k=1,2, \ldots, N_{*}$, such that

$$
\left|F(x)-\sum_{k=1}^{N_{*}} F_{k}(x)\right|=\mathcal{O}\left(|x|^{\beta}\right) \text { as } x \rightarrow 0 \text {, for all } \beta>\beta_{N_{*}} .
$$

In Assumption 4.1](ii), we conveniently write case (H1) as

$$
F(x) \sim \sum_{k=1}^{\infty} F_{k}(x)
$$

and case (H2) as

$$
F(x) \sim \sum_{k=1}^{N_{*}} F_{k}(x) .
$$

The following remarks on Assumption 4.1 are in order.

(a) Applying (2.4) and (2.5) to each function $F_{k}$, one has

$$
F_{k}(0)=0, \quad\left\|F_{k}\right\|_{\mathcal{H}_{\beta_{k}}}<\infty, \text { and }\left|F_{k}(x)\right| \leq\left\|F_{k}\right\|_{\mathcal{H}_{\beta_{k}}}|x|^{\beta_{k}} \text { for all } x \in \mathbb{R}^{d} .
$$

Hence, (4.1) indicates that the remainder $F(x)-\sum_{k=1}^{N} F_{k}(x)$ between $F(x)$ and its approximate sum $\sum_{k=1}^{N} F_{k}(x)$ is small, as $x \rightarrow 0$, of a higher order (of $|x|$ ) than that in the approximate sum $\sum_{k=1}^{N} F_{k}(x)$.

(b) With functions $F_{k}$ 's as in (H2) of Assumption 4.1, if $F(x)=\sum_{k=1}^{N_{*}} F_{k}(x)$, then $F$ satisfies (4.4). For the relation between (4.3) and (4.4), see Remark 4.4 below.

(c) By the remark (e) after Definition 2.2, if $F$ is a $C^{\infty}$-vector field on the entire space $\mathbb{R}^{d}$ with $F(0)=0$ and $F^{\prime}(0)=0$, then $F$ satisfies Assumption 4.1 with the right-hand side of (4.3) is simply the Taylor expansion of $F(x)$ about the origin. 
(d) Note that we do not require the convergence of the formal series on the right-hand side of (4.3). Even when the convergence occurs, the limit is not necessarily the function $F$. For instance, if $h: \mathbb{R}^{d} \rightarrow \mathbb{R}^{d}$ satisfies $|x|^{-\alpha} h(x) \rightarrow 0$ as $x \rightarrow 0$ for all $\alpha>0$, then $F$ and $F+h$ have the same expansion (4.3).

(e) The class of functions F's that satisfy Assumption 4.1 contains much more than smooth vector fields, see section 6 below.

By Assumption 4.1, for each $N \in \mathbb{N}$ in case of (4.3), or $N \in \mathbb{N} \cap\left[1, N_{*}\right]$ in case of (4.4), there is $\varepsilon_{N}>0$ such that

$$
\left|F(x)-\sum_{k=1}^{N} F_{k}(x)\right|=\mathcal{O}\left(|x|^{\beta_{N}+\varepsilon_{N}}\right) \text { as } x \rightarrow 0 .
$$

Note from (4.5) with $N=1$ that, as $x \rightarrow 0$,

$$
|F(x)| \leq\left|F_{1}(x)\right|+\left|F(x)-F_{1}(x)\right| \leq\left\|F_{1}\right\|_{\mathcal{H}_{\beta_{1}}}|x|^{\beta_{1}}+\mathcal{O}\left(|x|^{\beta_{1}+\varepsilon_{1}}\right)=\mathcal{O}\left(|x|^{\beta_{1}}\right) .
$$

Thus, there exist numbers $c_{*}, \varepsilon_{*}>0$ such that

$$
|F(x)| \leq c_{*}|x|^{\beta_{1}} \quad \forall x \in \mathbb{R}^{d} \text { with }|x|<\varepsilon_{*} .
$$

By property (4.6) and Assumption 4.1, function F satisfies conditions in Assumption 3.1. Therefore, the facts about trivial and non-trivial solutions in section 3 still applies to equation (1.4), and Theorem 3.3 holds true for solutions of (1.4).

Hereafter, $y(t)$ is a non-trivial, decaying solution of (1.4).

Let eigenvalue $\lambda_{*}=\lambda_{n_{0}}$ and its corresponding eigenvector $\xi_{*}$ be as in Theorem 3.3. It follow (3.14) that

$$
|y(t)|=\mathcal{O}\left(e^{-\lambda_{*} t}\right) .
$$

To describe the exponential rates in a possible asymptotic expansion of solution $y(t)$ we use the following sets $\widetilde{S}$ and $S$.

Definition 4.2. We define a set $\widetilde{S} \subset[0, \infty)$ as follows.

In the case of (4.3), let $\alpha_{k}=\beta_{k}-1>0$ for $k \in \mathbb{N}$, and

$$
\begin{gathered}
\widetilde{S}=\left\{\sum_{k=n_{0}}^{d_{*}} m_{k}\left(\lambda_{k}-\lambda_{*}\right)+\sum_{j=1}^{\infty} z_{j} \alpha_{j} \lambda_{*}: m_{k}, z_{j} \in \mathbb{Z}_{+},\right. \\
\text {with } \left.z_{j}>0 \text { for only finitely many } j \text { 's }\right\} .
\end{gathered}
$$

In the case of (4.4), let $\alpha_{k}=\beta_{k}-1>0$ for $k=1,2, \ldots, N_{*}$, and

$$
\widetilde{S}=\left\{\sum_{k=n_{0}}^{d_{*}} m_{k}\left(\lambda_{k}-\lambda_{*}\right)+\sum_{j=1}^{N_{*}} z_{j} \alpha_{j} \lambda_{*}: m_{k}, z_{j} \in \mathbb{Z}_{+}\right\} .
$$

In both cases, the set $\widetilde{S}$ has countably, infinitely many elements. Arrange $\widetilde{S}$ as a sequence $\left(\widetilde{\mu}_{n}\right)_{n=1}^{\infty}$ of non-negative and strictly increasing numbers. Set

$$
\mu_{n}=\widetilde{\mu}_{n}+\lambda_{*} \text { for } n \in \mathbb{N} \text {, and define } S=\left\{\mu_{n}: n \in \mathbb{N}\right\} \text {. }
$$

The set $\widetilde{S}$ has the following elementary properties. 
(a) For $n_{0} \leq \ell \leq d_{*}$, by choose $m_{k}=\delta_{k \ell}$, and $z_{j}=0$ for all $j$ in (4.8) or (4.9), we have $\lambda_{\ell}-\lambda_{*} \in \widetilde{S}$. Hence,

$$
\lambda_{\ell} \in S \text { for all } \ell=n_{0}, n_{0}+1, \ldots, d_{*} .
$$

(b) Clearly, $\widetilde{\mu}_{1}=0$ and $\mu_{1}=\lambda_{*}$. The numbers $\mu_{n}$ 's are positive and strictly increasing. Also,

$$
\widetilde{\mu}_{n} \rightarrow \infty \text { and } \mu_{n} \rightarrow \infty \text { as } n \rightarrow \infty .
$$

(c) For all $x, y \in \widetilde{S}$ and $k \in \mathbb{N}$, one has

$$
x+y, x+\alpha_{k} \lambda_{*} \in \widetilde{S} .
$$

As a consequence of (4.13), one has

$$
\widetilde{\mu}_{n}+\alpha_{k} \lambda_{*} \geq \widetilde{\mu}_{n+1} \text { for all } n, k \text {. }
$$

Let $r \in \mathbb{N}$ and $s \in \mathbb{Z}_{+}$. Since $F_{r}$ is a $C^{\infty}$-function in a neighborhood of $\xi_{*} \neq 0$, we have the following Taylor's expansion, for any $h \in \mathbb{R}^{d}$,

$$
F_{r}\left(\xi_{*}+h\right)=\sum_{m=0}^{s} \frac{1}{m !} D^{m} F_{r}\left(\xi_{*}\right) h^{(m)}+g_{r, s}(h),
$$

where $D^{m} F_{r}\left(\xi_{*}\right)$ is the $m$-th order derivative of $F_{r}$ at $\xi_{*}$, and

$$
g_{r, s}(h)=\mathcal{O}\left(|h|^{s+1}\right) \text { as } h \rightarrow 0 .
$$

For $m \geq 0$, denote

$$
\mathcal{F}_{r, m}=\frac{1}{m !} D^{m} F_{r}\left(\xi_{*}\right)
$$

When $m=0$, (4.17) reads as $\mathcal{F}_{r, 0}=F_{r}\left(\xi_{*}\right)$. When $m \geq 1, \mathcal{F}_{r, m}$ is an $m$-linear mapping from $\left(\mathbb{R}^{d}\right)^{m}$ to $\mathbb{R}^{d}$.

By (2.9), one has, for any $r, m \geq 1$, and $y_{1}, y_{2}, \ldots, y_{m} \in \mathbb{R}^{d}$, that

$$
\left|\mathcal{F}_{r, m}\left(y_{1}, y_{2}, \ldots, y_{m}\right)\right| \leq\left\|\mathcal{F}_{r, m}\right\| \cdot\left|y_{1}\right| \cdot\left|y_{2}\right| \cdots\left|y_{m}\right| \cdot
$$

For our convenience, we write inequality (4.18) even when $m=0$ with $\left\|\mathcal{F}_{r, 0}\right\| \stackrel{\text { def }}{=}\left|F_{r}\left(\xi_{*}\right)\right|$. Our main result is the following theorem.

Theorem 4.3. There exist polynomials $q_{n}: \mathbb{R} \rightarrow \mathbb{R}^{d}$ such that $y(t)$ has an asymptotic expansion, in the sense of Definition 2.1.

$$
y(t) \sim \sum_{n=1}^{\infty} q_{n}(t) e^{-\mu_{n} t} \text { in } \mathbb{R}^{d}
$$

where $\mu_{n}$ 's are defined in Definition 4.2, and $q_{n}(t)$ satisfies, for any $n \geq 1$,

$$
q_{n}^{\prime}+\left(A-\mu_{n} I_{d}\right) q_{n}=\mathcal{J}_{n} \stackrel{\text { def }}{=} \sum_{\substack{r \geq 1, m \geq 0, k_{1}, k_{2}, \ldots, k_{m} \geq 2, \sum_{j=1}^{m} \widetilde{\mu}_{k_{j}}+\alpha_{r} \lambda_{*}=\widetilde{\mu}_{n}}} \mathcal{F}_{r, m}\left(q_{k_{1}}, q_{k_{2}}, \ldots, q_{k_{m}}\right) \text { in } \mathbb{R}
$$

We clarify the notation in Theorem 4.3. 
(a) In case of assumption (4.3), the index $r$ in $\mathcal{J}_{n}$ is taken over the whole set $\mathbb{N}$. In case of assumption (4.4), the index $r$ in $\mathcal{J}_{n}$ is restricted to $1,2, \ldots, N_{*}$, thus, we explicitly have

$$
\mathcal{J}_{n}=\sum_{r=1}^{N_{*}} \sum_{\substack{m \geq 0 \\ \sum_{j=1}^{m}, k_{1}, k_{2}, \ldots, k_{m} \geq 2, \widetilde{\mu}_{k_{j}}+\alpha_{r} \lambda_{*}=\widetilde{\mu}_{n}}} \mathcal{F}_{r, m}\left(q_{k_{1}}, q_{k_{2}}, \ldots, q_{k_{m}}\right) .
$$

(b) When $m=0$, the terms $q_{k_{j}}$ 's in $\mathcal{J}_{n}$ are not needed, see the explanation after (4.17), hence the condition $k_{j} \geq 2$ is ignored, and the corresponding terms in $\mathcal{J}_{n}$ becomes

$$
\sum F_{r}\left(\xi_{*}\right) \text { for } \alpha_{r} \lambda_{*}=\widetilde{\mu}_{n} \text {, that is, } \beta_{r} \lambda_{*}=\mu_{n} \text {. }
$$

Thus, we rewrite (4.20) more explicitly, by considering $m=0$ and $m \geq 1$ for $\mathcal{J}_{n}$, as

$$
q_{n}^{\prime}+\left(A-\mu_{n} I_{d}\right) q_{n}=\sum_{r \geq 1, \alpha_{r} \lambda_{*}=\widetilde{\mu}_{n}} F_{r}\left(\xi_{*}\right)+\sum_{\substack{r \geq 1, m \geq 1, k_{1}, k_{2}, \ldots, k_{m} \geq 2, \sum_{j=1}^{m} \widetilde{\mu}_{k_{j}}+\alpha_{r} \lambda_{*}=\widetilde{\mu}_{n}}} \mathcal{F}_{r, m}\left(q_{k_{1}}, q_{k_{2}}, \ldots, q_{k_{m}}\right) .
$$

Note, in (4.22), that such an index $r$ may or may not exists. In the latter case, the term is understood to be zero. In the former case, $r$ is uniquely determined and we have only one term.

(c) When $n=1$, we have $\widetilde{\mu}_{1}=0$, and there are no indices satisfying constraints for the sum in $\mathcal{J}_{1}$. Hence $\mathcal{J}_{1}=0$, and (4.20) becomes

$$
q_{1}^{\prime}+\left(A-\mu_{1} I_{d}\right) q_{1}=0,
$$

(d) Consider $n=2$. If $m \geq 1$, then, for the second sum on the right-hand side of (4.23), one has at least $\widetilde{\mu}_{k_{1}} \geq \widetilde{\mu}_{2}$. Hence $\widetilde{\mu}_{k_{j}}+\alpha_{r} \lambda_{*}>\mu_{k_{1}} \geq \widetilde{\mu}_{2}$. Therefore, the last condition for the indices in the second sum on the right-hand side of (4.23) is not met. Thus, (4.23) becomes

$$
q_{2}^{\prime}+\left(A-\mu_{2} I_{d}\right) q_{2}=\mathcal{J}_{2}=\sum_{r \geq 1, \alpha_{r} \lambda_{*}=\widetilde{\mu}_{2}} F_{r}\left(\xi_{*}\right)=\sum_{r \geq 1, \beta_{r} \lambda_{*}=\mu_{2}} F_{r}\left(\xi_{*}\right) .
$$

(e) We verify that the sum in $\mathcal{J}_{n}$ is a finite sum.

Let $n \geq 2$. Firstly, the indices in the sum of $\mathcal{J}_{n}$ satisfy

$$
\widetilde{\mu}_{n}=\sum_{j=1}^{m} \widetilde{\mu}_{k_{j}}+\alpha_{r} \lambda_{*} \geq \alpha_{r} \lambda_{*}=\alpha_{r} \mu_{1}
$$

Then

$$
\alpha_{r} \leq \widetilde{\mu}_{n} / \mu_{1}
$$

Secondly, for $m \geq 1$, one has

$$
\widetilde{\mu}_{n}=\sum_{j=1}^{m} \widetilde{\mu}_{k_{j}}+\alpha_{r} \lambda_{*}>\sum_{j=1}^{m} \mu_{k_{j}} \geq m \widetilde{\mu}_{2}
$$

which yields

$$
m<\widetilde{\mu}_{n} / \widetilde{\mu}_{2} \text {. }
$$

Note that condition (4.27) is not met for $n=2$ and $m \geq 1$.

Thirdly, $\widetilde{\mu}_{n}=\sum_{j=1}^{m} \widetilde{\mu}_{k_{j}}+\alpha_{r} \lambda_{*}>\widetilde{\mu}_{k_{j}}$, which yields

$$
k_{j}<n \text {. }
$$


Hence, the terms $q_{k_{j}}$ 's in (4.20) come from previous steps.

By (4.25), (4.26), (4.27), the sum in $\mathcal{J}_{n}$ is over only finitely many $r$ 's, $m$ 's and $k_{j}$ 's.

(f) For $n \geq 2$, suppose $r^{*}, m^{*}, k^{*}$ are non-negative integers such that

$$
\alpha_{r^{*}} \geq \widetilde{\mu}_{n} / \mu_{1}, m^{*} \geq \widetilde{\mu}_{n} / \widetilde{\mu}_{2}, k^{*} \geq n-1 .
$$

Then $\mathcal{J}_{n}$ can be equivalently written as

$$
\mathcal{J}_{n}=\sum_{r=1}^{r^{*}} \sum_{m=0}^{m^{*}} \sum_{\substack{2 \leq k_{1}, k_{2}, \ldots, k_{m} \leq k^{*}, \sum_{j=1}^{m} \widetilde{\mu}_{k_{j}}+\alpha_{r} \mu_{1}=\widetilde{\mu}_{n}}} \mathcal{F}_{r, m}\left(q_{k_{1}}, q_{k_{2}}, \ldots, q_{k_{m}}\right) \text {. }
$$

Clearly, the right-hand side of (4.29) is a part of the sum in $\mathcal{J}_{n}$, and the converse is also true thanks to (4.25), (4.26) and (4.27) above. Thus, the sums on both sides of (4.29) are the same.

(g) In case of (4.4) and $n \geq 2, \mathcal{J}_{n}$ is given by (4.21), and relation (4.29) under condition (4.28) can be recast as

$$
\mathcal{J}_{n}=\sum_{r=1}^{N_{*}} \sum_{m=0}^{m^{*}} \sum_{\substack{2 \leq k_{1}, k_{2}, \ldots, k_{m} \leq k^{*} \\ \sum_{j=1}^{m}, \widetilde{\mu}_{k_{j}}+\alpha_{r} \mu_{1}=\widetilde{\mu}_{n}}} \mathcal{F}_{r, m}\left(q_{k_{1}}, q_{k_{2}}, \ldots, q_{k_{m}}\right),
$$

for any non-negative integers $m^{*}, k^{*}$ satisfying

$$
m^{*} \geq \widetilde{\mu}_{n} / \widetilde{\mu}_{2} \text { and } k^{*} \geq n-1 \text {. }
$$

We are ready to prove Theorem 4.3 now.

Proof of Theorem 4.3. We will prove for the case (4.3) first, and then make necessary changes for the case (4.4) later.

Part A: Proof for the case of (4.3). For any $N \in \mathbb{N}$, we denote by $\left(\mathcal{T}_{N}\right)$ the following statement: There exist $\mathbb{R}^{d}$-valued polynomials $q_{1}(t), q_{2}(t), \ldots, q_{N}(t)$ such that equation (4.20) holds true for $n=1,2, \ldots, N$, and

$$
\left|y(t)-\sum_{n=1}^{N} q_{n}(t) e^{-\mu_{n} t}\right|=\mathcal{O}\left(e^{-\left(\mu_{N}+\delta_{N}\right) t}\right) \quad \text { as } t \rightarrow \infty,
$$

for some $\delta_{N}>0$.

We will prove $\left(\mathcal{T}_{N}\right)$ for all $N \in \mathbb{N}$ by induction in $N$.

First step $(\mathrm{N}=1)$. By Theorem 3.3 and the fact $\mu_{1}=\lambda_{*}$, the statement $\left(\mathcal{T}_{1}\right)$ is true with $q_{1}(t)=\xi_{*}$ for all $t \in \mathbb{R}$, and some $\delta_{1}>0$.

Induction step. Let $N \geq 1$. Suppose there are polynomials $q_{n}$ 's for $1 \leq n \leq N$ such that the statement $\left(\mathcal{T}_{N}\right)$ holds true.

For $n=1, \ldots, N$, let $y_{n}(t)=q_{n}(t) e^{-\mu_{n} t}, u_{n}(t)=y(t)-\sum_{k=1}^{n} y_{k}(t)$. By induction hypotheses, the polynomials $q_{n}$ 's satisfy (4.24), (4.20) and

$$
u_{N}(t)=\mathcal{O}\left(e^{-\left(\mu_{N}+\delta_{N}\right) t}\right) .
$$


Let $w_{N}(t)=e^{\mu_{N+1} t} u_{N}(t)$. We derive the differential equation for $w_{N}(t)$.

$$
\begin{aligned}
w_{N}^{\prime}-\mu_{N+1} w_{N} & =u_{N}^{\prime} e^{\mu_{N+1} t}=\left(y^{\prime}-\sum_{k=1}^{N} y_{k}^{\prime}\right) e^{\mu_{N+1} t}=\left(-A y+F(y)-\sum_{k=1}^{N} y_{k}^{\prime}\right) e^{\mu_{N+1} t} \\
& =\left(-A u_{N}-\sum_{k=1}^{N} A y_{k}+F(y)-\sum_{k=1}^{N} y_{k}^{\prime}\right) e^{\mu_{N+1} t} .
\end{aligned}
$$

Thus

$$
w_{N}^{\prime}+\left(A-\mu_{N+1} I_{d}\right) w_{N}=e^{\mu_{N+1} t} F(y)-e^{\mu_{N+1} t} \sum_{k=1}^{N}\left(A y_{k}+y_{k}^{\prime}\right) .
$$

By (4.12), we can choose a number $r_{*} \in \mathbb{N}$ such that

$$
\beta_{r_{*}} \geq \mu_{N+1} / \mu_{1} \text {, which is equivalent to } \alpha_{r_{*}} \geq \widetilde{\mu}_{N+1} / \mu_{1} \text {. }
$$

By (4.5), one has

$$
F(x)=\sum_{r=1}^{r_{*}} F_{r}(x)+\mathcal{O}\left(|x|^{\beta_{r_{*}}+\varepsilon_{r_{*}}}\right) \text { as } x \rightarrow 0 .
$$

Using (4.36) with $x=y(t)$ and utilizing property (4.7), we write the first term on the right-hand side of (4.34) as

$e^{\mu_{N+1} t} F(y(t))=e^{\mu_{N+1} t} \sum_{r=1}^{r_{*}} F_{r}(y(t))+e^{\mu_{N+1} t} \mathcal{O}\left(|y(t)|^{\beta_{r_{*}}+\varepsilon_{r_{*}}}\right)=E(t)+e^{\mu_{N+1} t} \mathcal{O}\left(e^{-\lambda_{*}\left(\beta_{r_{*}}+\varepsilon_{r_{*}}\right) t}\right)$, where

$$
E(t)=e^{\mu_{N+1} t} \sum_{r=1}^{r_{*}} F_{r}(y(t))
$$

Because of condition for $\beta_{r_{*}}$ in (4.35), we then have

$$
e^{\mu_{N+1} t} F(y(t))=E(t)+\mathcal{O}\left(e^{-\widetilde{\delta}_{N} t}\right) \text {, where } \widetilde{\delta}_{N}=\lambda_{*} \varepsilon_{r_{*}} .
$$

The term $\sum_{r=1}^{r_{*}} F_{r}(y)$ in (4.37) will be calculated as below. For $k=1, \ldots, N$, denote

$$
\widetilde{y}_{k}(t)=y_{k}(t) e^{\lambda_{*} t}=q_{k}(t) e^{-\widetilde{\mu}_{k} t} \text { and } \widetilde{u}_{k}(t)=u_{k}(t) e^{\lambda_{*} t} .
$$

When $2 \leq k \leq N$, one has

$$
\widetilde{y}_{k}(t)=q_{k}(t) e^{-\widetilde{\mu}_{k} t}=\mathcal{O}\left(e^{-\left(\widetilde{\mu}_{k}-\varepsilon\right) t}\right) \text { for any } \varepsilon \in\left(0, \widetilde{\mu}_{k}\right) .
$$

By (4.33),

$$
\widetilde{u}_{N}(t)=u_{N}(t) e^{\lambda_{*} t}=\mathcal{O}\left(e^{-\left(\widetilde{\mu}_{N}+\delta_{N}\right) t}\right) .
$$

Also, from $\left(\mathcal{T}_{1}\right)$, we similarly have

$$
\widetilde{u}_{1}(t)=u_{1}(t) e^{\lambda_{*} t}=\mathcal{O}\left(e^{-\delta_{1} t}\right) .
$$

Then

$$
F_{r}(y(t))=F_{r}\left(y_{1}+u_{1}\right)=F_{r}\left(e^{-\lambda_{*} t}\left(\xi_{*}+\widetilde{u}_{1}\right)\right)=e^{-\beta_{r} \lambda_{*} t} F_{r}\left(\xi_{*}+\widetilde{u}_{1}\right) .
$$

Let $s_{*} \in \mathbb{N}$ satisfy

$$
s_{*} \delta_{1}+\beta_{1} \lambda_{*} \geq \mu_{N+1} \text { and } s_{*} \geq \widetilde{\mu}_{N+1} / \widetilde{\mu}_{2} \text {. }
$$


By Taylor's expansion (4.15) with $s=s_{*}$, using the notation in (4.17),

$$
F_{r}\left(\xi_{*}+\widetilde{u}_{1}\right)=\sum_{m=0}^{s_{*}} \mathcal{F}_{r, m} \widetilde{u}_{1}^{(m)}+g_{r, s_{*}}\left(\widetilde{u}_{1}\right) .
$$

It follows (4.42) and (4.44) that

$$
F_{r}(y(t))=e^{-\beta_{r} \lambda_{*} t}\left(F_{r}\left(\xi_{*}\right)+\sum_{m=1}^{s_{*}} \mathcal{F}_{r, m} \widetilde{u}_{1}^{(m)}\right)+e^{-\beta_{r} \lambda_{*} t} g_{r, s_{*}}\left(\widetilde{u}_{1}\right) .
$$

The terms in (4.45) are further calculated as follows.

For the last term in (4.45), by using (4.16), (4.41) and the first condition in (4.43), we find that

$$
\begin{aligned}
e^{-\beta_{r} \lambda_{*} t} g_{r, s_{*}}\left(\widetilde{u}_{1}\right) & =e^{-\beta_{r} \lambda_{*} t} \mathcal{O}\left(\left|\widetilde{u}_{1}(t)\right|^{s_{*}+1}\right)=e^{-\beta_{r} \lambda_{*} t} \mathcal{O}\left(e^{-\delta_{1}\left(s_{*}+1\right) t}\right) \\
& =\mathcal{O}\left(e^{-\left(\beta_{1} \lambda_{*}+\delta_{1} s_{*}+\delta_{1}\right) t}\right)=\mathcal{O}\left(e^{-\left(\mu_{N+1}+\delta_{1}\right) t}\right) .
\end{aligned}
$$

For the remaining terms on the right-hand side of (4.45), we write

$$
\begin{aligned}
\mathcal{F}_{r, m} \widetilde{u}_{1}^{(m)} & =\mathcal{F}_{r, m}\left(\sum_{k=2}^{N} \widetilde{y}_{k}+\widetilde{u}_{N}\right)^{(m)}=\mathcal{F}_{r, m}\left(\sum_{k=2}^{N} \widetilde{y}_{k}+\widetilde{u}_{N}, \sum_{k=2}^{N} \widetilde{y}_{k}+\widetilde{u}_{N}, \ldots, \sum_{k=2}^{N} \widetilde{y}_{k}+\widetilde{u}_{N}\right) \\
& =\mathcal{F}_{r, m}\left(\sum_{k=2}^{N} \widetilde{y}_{k}\right)^{(m)}+\sum_{\text {finitely many }} \mathcal{F}_{r, m}\left(z_{1}, \ldots, z_{N}\right) .
\end{aligned}
$$

Note, in the case $N=1$, that the sum $\sum_{k=2}^{N} \widetilde{y}_{k}$ and, hence, the term $\mathcal{F}_{r, m}\left(\sum_{k=2}^{N} \widetilde{y}_{k}\right)^{(m)}$ are not present in the calculations in (4.47). In the last sum of (4.47), each $z_{1}, \ldots, z_{N}$ is either $\sum_{k=2}^{N} \widetilde{y}_{k}$ or $\widetilde{u}_{N}$, and at least one of $z_{j}$ 's must be $\widetilde{u}_{N}$. By inequality (4.18), estimate (4.39) for $\widetilde{y}_{k}$, and estimates (4.40), (4.41) for $\widetilde{u}_{N}$, we have

$$
\left|\mathcal{F}_{r, m}\left(z_{1}, \ldots, z_{N}\right)\right| \leq\left\|\mathcal{F}_{r, m}\right\| \cdot\left|z_{1}\right| \ldots\left|z_{N}\right|=\mathcal{O}\left(\left|\widetilde{u}_{N}\right|\right)=\mathcal{O}\left(e^{-\left(\widetilde{\mu}_{N}+\delta_{N}\right) t}\right) .
$$

Therefore,

$$
\begin{aligned}
\sum_{m=0}^{s_{*}} \mathcal{F}_{r, m} \widetilde{u}_{1}^{(m)} & =F_{r}\left(\xi_{*}\right)+\sum_{m=1}^{s_{*}} \mathcal{F}_{r, m}\left(\sum_{k=2}^{N} \widetilde{y}_{k}\right)^{(m)}+\mathcal{O}\left(e^{-\left(\widetilde{\mu}_{N}+\delta_{N}\right) t}\right) \\
& =\sum_{m=0}^{s_{*}} \sum_{k_{1}, \ldots, k_{m} \geq 2}^{N} \mathcal{F}_{r, m}\left(\widetilde{y}_{k_{1}}, \widetilde{y}_{k_{2}}, \ldots, \widetilde{y}_{k_{m}}\right)+\mathcal{O}\left(e^{-\left(\widetilde{\mu}_{N}+\delta_{N}\right) t}\right) \\
& =\sum_{m=0}^{s_{*}} \sum_{k_{1}, \ldots, k_{m} \geq 2}^{N} e^{-t \sum_{j=1}^{m} \widetilde{\mu}_{k_{j}}} \mathcal{F}_{r, m}\left(q_{k_{1}}, q_{k_{2}}, \ldots, q_{k_{m}}\right)+\mathcal{O}\left(e^{-\left(\widetilde{\mu}_{N}+\delta_{N}\right) t}\right) .
\end{aligned}
$$

Thus,

$$
\begin{aligned}
e^{-\beta_{r} \lambda_{*} t} \sum_{m=0}^{s_{*}} \mathcal{F}_{r, m} \widetilde{u}_{1}^{(m)}= & \sum_{m=0}^{s_{*}} \sum_{k_{1}, \ldots, k_{m}=2}^{N} e^{-t\left(\sum_{j=1}^{m} \widetilde{\mu}_{k_{j}}+\beta_{r} \lambda_{*}\right)} \mathcal{F}_{r, m}\left(q_{k_{1}}, q_{k_{2}}, \ldots, q_{k_{m}}\right) \\
& +\mathcal{O}\left(e^{-\left(\widetilde{\mu}_{N}+\beta_{r} \lambda_{*}+\delta_{N}\right) t}\right)
\end{aligned}
$$


Again, in the case $N=1$, the last double summation has only one term corresponding to $m=0$, which is $F_{r}\left(\xi_{*}\right)$.

Using property (4.14), we have

$$
\widetilde{\mu}_{N}+\beta_{r} \lambda_{*}+\delta_{N}=\widetilde{\mu}_{N}+\alpha_{r} \lambda_{*}+\lambda_{*}+\delta_{N} \geq \widetilde{\mu}_{N+1}+\lambda_{*}+\delta_{N}=\mu_{N+1}+\delta_{N} .
$$

Hence, the last term in (4.48) can be estimated as

$$
\mathcal{O}\left(e^{-\left(\widetilde{\mu}_{N}+\beta_{r} \lambda_{*}+\delta_{N}\right) t}\right)=\mathcal{O}\left(e^{-\left(\mu_{N+1}+\delta_{N}\right) t}\right) .
$$

Therefore, by formula of $E(t)$ in (4.37), and (4.45), (4.46), (4.48), (4.49), we have

$$
E(t)=e^{\mu_{N+1} t}\left(J+\mathcal{O}\left(e^{-\left(\mu_{N+1}+\delta_{N}\right) t}\right)+\mathcal{O}\left(e^{-\left(\mu_{N+1}+\delta_{1}\right) t}\right)\right)=e^{\mu_{N+1} t} J+\mathcal{O}\left(e^{-\min \left\{\delta_{1}, \delta_{N}\right\} t}\right),
$$

where

$$
J=\sum_{r=1}^{r_{*}} \sum_{m=0}^{s_{*}} \sum_{k_{1}, \ldots, k_{m} \geq 2}^{N} e^{-t\left(\sum_{j=1}^{m} \widetilde{\mu}_{k_{j}}+\beta_{r} \lambda_{*}\right)} \mathcal{F}_{r, m}\left(q_{k_{1}}, q_{k_{2}}, \ldots, q_{k_{m}}\right) .
$$

Denote $\mu=\widetilde{\mu}_{k_{1}}+\ldots+\widetilde{\mu}_{k_{m}}+\alpha_{r} \lambda_{*}$. When $m=0$, one has $\mu=\alpha_{r} \lambda_{*}$, which belongs to $\widetilde{S}$. When $m \geq 1$, by property (4.13), $\mu$ also belongs to $\widetilde{S}$. Clearly, $\mu>0=\widetilde{\mu}_{1}$. Thus, in both cases of $m$, the number $\mu$ must equal $\widetilde{\mu}_{p}$ for a unique $p \geq 2$. Because of the indices $r, m, k_{1}, \ldots, k_{m}$ being finitely many, there are only finitely many such numbers $p$ 's. Thus, there is $p_{*} \in \mathbb{N}$ such that any index $p$ above satisfies $p \leq p_{*}$. Hence, the exponent in (4.51) is

$$
\sum_{j=1}^{m} \widetilde{\mu}_{k_{j}}+\beta_{r} \lambda_{*}=\mu+\lambda_{*}=\widetilde{\mu}_{p}+\lambda_{*}=\mu_{p} \quad \text { for some integer } p \in\left[2, p_{*}\right] .
$$

Using index $p$ in (4.52), we can split the sum in $J$ into two parts corresponding to $p \leq N+1$ and $p \geq N+2$. We then write $J=S_{1}+S_{2}$, where

$$
\begin{aligned}
& S_{1}=\sum_{p=2}^{N+1} \sum_{r=1}^{r_{*}} \sum_{m=0}^{s_{*}} \sum_{\substack{2 \leq k_{1}, \ldots, k_{m} \leq N, \sum_{j=1}^{m} \widetilde{\mu}_{k_{j}}+\beta_{r} \lambda_{*}=\mu_{p}}} e^{-\mu_{p} t} \mathcal{F}_{r, m}\left(q_{k_{1}}, q_{k_{2}}, \ldots, q_{k_{m}}\right), \\
& S_{2}=\sum_{p=N+2}^{p_{*}} \sum_{r=1}^{r_{*}} \sum_{m=0}^{s_{*}} \sum_{\substack{2 \leq k_{1}, \ldots, k_{m} \leq N, \sum_{j=1}^{m}, \ldots, \mu_{k_{j}}+\beta_{r} \lambda_{*}=\mu_{p}}} e^{-\mu_{p} t} \mathcal{F}_{r, m}\left(q_{k_{1}}, q_{k_{2}}, \ldots, q_{k_{m}}\right) .
\end{aligned}
$$

We re-write $S_{1}=\sum_{k=2}^{N+1} e^{-\mu_{k} t} J_{k}$, where

$$
J_{k}=\sum_{r=1}^{r_{*}} \sum_{m=0}^{s_{*}} \sum_{\substack{2 \leq k_{1}, \ldots, k_{m} \leq N, \sum_{j=1}^{m} \widetilde{\mu}_{k_{j}}+\beta_{r} \lambda_{*}=\mu_{k}}} \mathcal{F}_{r, m}\left(q_{k_{1}}, q_{k_{2}}, \ldots, q_{k_{m}}\right) \text { for } k=1,2, \ldots, N+1 .
$$

We estimate $S_{2}$. Set $\delta_{N}^{\prime}=\min \left\{\widetilde{\delta}_{N}, \delta_{1}, \delta_{N},\left(\mu_{N+2}-\mu_{N+1}\right) / 2\right\}>0$. Using inequality (4.18) to estimate $\left|\mathcal{F}_{r, m}\left(q_{k_{1}}, q_{k_{2}}, \ldots, q_{k_{m}}\right)\right|$, and recalling that $q_{k_{j}}$ 's are polynomials in $t$, we have

$$
\left|\mathcal{F}_{r, m}\left(q_{k_{1}}, q_{k_{2}}, \ldots, q_{k_{m}}\right)\right| \leq\left\|\mathcal{F}_{r, m}\right\| \cdot\left|q_{k_{1}}\right| \cdot\left|q_{k_{2}}\right| \ldots\left|q_{k_{m}}\right|=\mathcal{O}\left(e^{\delta_{N}^{\prime} t}\right) .
$$

For $e^{-\mu_{p} t}$, we use $\mu_{p} \geq \mu_{N+2}$, and obtain

$$
S_{2}=\mathcal{O}\left(e^{-\mu_{N+2} t} e^{\delta_{N}^{\prime} t}\right)=\mathcal{O}\left(e^{-\left(\mu_{N+1}+\delta_{N}^{\prime}\right) t}\right) .
$$


Combining the above calculations from (4.50) to (4.54) gives

$$
E(t)=e^{\mu_{N+1} t} \sum_{k=2}^{N+1} e^{-\mu_{k} t} J_{k}+\mathcal{O}\left(e^{-\delta_{N}^{\prime} t}\right) .
$$

Thus, by (4.34), (4.38) and (4.55),

$$
w_{N}^{\prime}+\left(A-\mu_{N+1} I_{d}\right) w_{N}=\left(\sum_{k=2}^{N+1} e^{-\mu_{k} t} J_{k}-\sum_{k=1}^{N}\left(A y_{k}+y_{k}^{\prime}\right)\right) e^{\mu_{N+1} t}+\mathcal{O}\left(e^{-\delta_{N}^{\prime} t}\right) .
$$

Using the fact $A y_{k}+y_{k}^{\prime}=e^{-\mu_{k} t}\left(q_{k}^{\prime}+\left(A-\mu_{k} I_{d}\right) q_{k}\right)$, for $k=1,2, \ldots, N$, we deduce

$$
w_{N}^{\prime}+\left(A-\mu_{N+1} I_{d}\right) w_{N}=-e^{\mu_{N+1} t} \sum_{k=1}^{N} e^{-\mu_{k} t} \chi_{k}+J_{N+1}+\mathcal{O}\left(e^{-\delta_{N}^{\prime} t}\right),
$$

where

$$
\chi_{1}=q_{1}^{\prime}+\left(A-\mu_{1} I_{d}\right) q_{1}, \quad \chi_{k}=q_{k}^{\prime}+\left(A-\mu_{k} I_{d}\right) q_{k}-J_{k} \text { for } 2 \leq k \leq N .
$$

We already know $\chi_{1}=0$. Let us focus on the sum $\sum_{k=1}^{N} e^{-\mu_{k} t} \chi_{k}$ on the right-hand side of (4.56). In case $N=1$, this sum is already zero.

Consider $N \geq 2$. Note that condition $\sum_{j=1}^{m} \widetilde{\mu}_{k_{j}}+\beta_{r} \lambda_{*}=\mu_{k}$ in formula (4.53) of $J_{k}$ is equivalent to $\sum_{j=1}^{m} \widetilde{\mu}_{k_{j}}+\alpha_{r} \lambda_{*}=\widetilde{\mu}_{k}$. Then, for each $k=1,2, \ldots, N+1$, by the virtue of relation (4.29) for $n=k \leq N+1, r^{*}=r_{*}, m^{*}=s_{*}$ and $k^{*}=N$, one has

$$
J_{k}=\mathcal{J}_{k} \text { for } k=1,2, \ldots, N+1 \text {. }
$$

Above, condition (4.28) is met thanks to the condition for $\alpha_{r_{*}}$ in (4.35), the second condition for $s_{*}$ in (4.43), and the fact $N \geq k-1$.

Thanks to (4.57) and the induction hypothesis, $\chi_{k}=0$ for $2 \leq k \leq N$. Hence, (4.56) becomes

$$
w_{N}^{\prime}+\left(A-\mu_{N+1} I_{d}\right) w_{N}=J_{N+1}+\mathcal{O}\left(e^{-\delta_{N}^{\prime} t}\right) .
$$

Note that $\mu_{N+1}>\mu_{1} \geq \lambda_{1}$. Let $\lambda_{i}$ is an eigenvalue of $A$ with $\lambda_{i}<\mu_{N+1}$. If $\lambda_{i} \leq \lambda_{n_{0}}=\mu_{1}$ then $\lambda_{i} \leq \mu_{N}$. If $\lambda_{i}>\lambda_{n_{0}}$, then, according to property (4.11), $\lambda_{i} \in S$, hence, by the constraint $\lambda_{i}<\mu_{N+1}$, we have $\lambda_{i} \leq \mu_{N}$. Therefore, in both cases

$$
e^{\left(\lambda_{i}-\mu_{N+1}\right) t}\left|w_{N}(t)\right|=e^{\lambda_{i} t}\left|u_{N}(t)\right|=e^{\lambda_{i} t} \mathcal{O}\left(e^{-\left(\mu_{N}+\delta_{N}\right) t}\right)=\mathcal{O}\left(e^{-\delta_{N} t}\right) .
$$

That is, condition (2.12) is satisfied.

Applying Lemma 2.4 to the equation (4.58), there exists polynomial $q_{N+1}: \mathbb{R} \rightarrow \mathbb{R}^{d}$ and a number $\delta_{N+1}>0$ such that

$$
\left|w_{N}(t)-q_{N+1}(t)\right|=\mathcal{O}\left(e^{-\delta_{N+1} t}\right) .
$$

Moreover $q_{N+1}(t)$ solves

$$
q_{N+1}^{\prime}+\left(A-\mu_{N+1} I_{d}\right) q_{N+1}=J_{N+1}=\mathcal{J}_{N+1},
$$

that is, equation (4.20) holds for $n=N+1$.

Multiplying (4.59) by $e^{-\mu_{N+1} t}$ gives

$$
\left|y(t)-\sum_{n=1}^{N+1} q_{n}(t) e^{-\mu_{n} t}\right|=\mathcal{O}\left(e^{-\left(\mu_{N+1}+\delta_{N+1}\right) t}\right),
$$

which proves (4.32) for $N:=N+1$. 
Hence the statement $\left(\mathcal{T}_{N+1}\right)$ holds true.

Conclusion for Part A. By the induction principle, the statement $\left(\mathcal{T}_{N}\right)$ holds true for all $N \in \mathbb{N}$. Note also that, the polynomials $\left(\mathcal{T}_{N+1}\right)$ are exactly the ones from $\left(\mathcal{T}_{N}\right)$. Hence, the polynomials $q_{n}$ 's exist for all $n \in \mathbb{N}$, for which $\left(\mathcal{T}_{N}\right)$ holds true for all $N \in \mathbb{N}$. Therefore, we obtain the desired expansion (4.19).

Part B: Proof for the case of (4.4). We follow the proof in Part A with the following adjustments. The number $r_{*}$ is simply $N_{*}$, and condition (4.35) for $r_{*}$ is not required anymore. All the sum $\sum_{r \geq 1}$ appearing in the proof that involves $F_{r}$ or $\mathcal{F}_{r, m}$ will be replaced with $\sum_{1 \leq r \leq N_{*}}$. From (4.36) to the end of the proof in Part A, positive number $\varepsilon_{r_{*}}$ is arbitrary, and number $\beta_{r_{*}}$ in calculations from (4.36) to (4.38) is replaced with any number $\beta_{*} \geq \mu_{N+1} / \mu_{1}$. Then (4.36) still holds true thanks to (4.2). We also take into account that $\mathcal{J}_{n}$ is given by (4.21), and one has relation (4.30) under condition (4.31). With these changes, the above proof in Part A goes through, and we obtain the desired statement for this case (4.4).

The proof of Theorem 4.3 is now complete.

Remark 4.4. Assume we have (4.4), then by adding more functions $F_{k}=0$ and numbers $\beta_{k}$ 's, for $k>N_{*}$, such that $\beta_{k}$ increases strictly to infinity, one can convert (4.4) into (4.3). (For example, one can take $\beta_{k}=\beta_{N_{*}}+k$ for $k>N_{*}$.) However, we did not use this fact in Part B of the proof of Theorem 4.3 above. The reason is to have simpler constructions of $\widetilde{S}$ and $q_{n}$ 's in (4.9) and (4.21) for the case (4.4), as opposed to (4.8) and (4.20) if it is converted to (4.3).

\section{EXTENDED RESULTS} $F$.

In this section, we extend Theorem 4.3 to the situations that require less of the function

First, we consider the case when the function $F$ in (1.4) only has a finite sum approximation. We will find a finite sum asymptotic approximation for decaying solutions of (1.4).

Assume function $F$ satisfies (i) and (H2) of Assumption 4.1 with (4.2) being replaced with

$$
\left|F(x)-\sum_{k=1}^{N_{*}} F_{k}(x)\right|=\mathcal{O}\left(|x|^{\beta_{N_{*}}+\bar{\varepsilon}}\right) \text { as } x \rightarrow 0 \text {, for some number } \bar{\varepsilon}>0 \text {. }
$$

Note that (5.1) is different from (4.2) due to the restriction of $\bar{\varepsilon}$. Also, we usually think of $\bar{\varepsilon}$ as a small number, but, in (5.1), it can be large. This happens when the remainder $F(x)-\sum_{k=1}^{N_{*}} F_{k}(x)$ may have very precise approximation, i.e., large $\bar{\varepsilon}$, but it does not have a homogeneous structure that we can take advantage of.

From (5.1), one can see that estimate (4.5) still holds for all $N \in \mathbb{N} \cap\left[1, N_{*}\right]$, where $\delta_{N}$ in any number in $\left(0, \beta_{N+1}-\beta_{N}\right)$ when $N<N_{*}$, and is $\bar{\varepsilon}$ when $N=N_{*}$. Consequently, (4.6) is still valid, and the facts and results in section 3 apply.

Let $y(t)$ be a non-trivial, decaying solution of (1.4). Applying Theorem 3.3, we have the first approximation (3.14).

For more precise approximations, define sets $\widetilde{S}$ and $S$ by (4.9) and (4.10), respectively.

Let $\bar{N} \in \mathbb{N}$ be defined by

$$
\bar{N}=\max \left\{N \in \mathbb{N}: \lambda_{*}\left(\beta_{N_{*}}+\bar{\varepsilon}\right)>\mu_{N}\right\} .
$$


From the definition of $\widetilde{S}$, we see that $\alpha_{N_{*}} \lambda_{*} \in \widetilde{S}$. Therefore, there exists a unique number $N^{\prime} \in \mathbb{N}$ such that $\alpha_{N_{*}} \lambda_{*}=\widetilde{\mu}_{N^{\prime}}$, which is equivalent to $\mu_{N^{\prime}}=\beta_{N_{*}} \lambda_{*}$. The last expression gives $\mu_{N^{\prime}}>\lambda_{*}=\mu_{1}$, thus, one must have $N^{\prime} \geq 2$. Note that $N^{\prime}$ belongs to the set on the right-hand side of (5.2), then $\bar{N} \geq N^{\prime} \geq 2$.

We obtain the finite approximation for decaying solutions under the assumption (5.1) as follows.

Theorem 5.1. There exist $\mathbb{R}^{d}$-valued polynomials $q_{n}(t)^{\prime}$ 's, for $1 \leq n \leq \bar{N}$, and a number $\delta>0$ such that

$$
\left|y(t)-\sum_{n=1}^{\bar{N}} q_{n}(t) e^{-\mu_{n} t}\right|=\mathcal{O}\left(e^{-\left(\mu_{\bar{N}}+\delta\right) t}\right),
$$

where each polynomial $q_{n}(t)$, for $1 \leq n \leq \bar{N}$, satisfies equation

$$
q_{n}^{\prime}+\left(A-\mu_{n} I_{d}\right) q_{n}=\sum_{r=1}^{N_{*}} \sum_{\substack{m \geq 0, k_{1}, k_{2}, \ldots, k_{m} \geq 2, \sum_{j=1}^{m} \widetilde{\mu}_{k_{j}}+\alpha_{r} \lambda_{*}=\widetilde{\mu}_{n}}} \mathcal{F}_{r, m}\left(q_{k_{1}}, q_{k_{2}}, \ldots, q_{k_{m}}\right) \text { in } \mathbb{R} .
$$

Proof. We follow Part A of the proof of Theorem 4.3, with some changes similar to those in Part B.

First, we take $r_{*}=N_{*}, 1 \leq r \leq N_{*}$ and replace $\varepsilon_{r_{*}}$ with number $\bar{\varepsilon}$ in (5.1).

Second, we replace condition (4.35) with $\lambda_{*}\left(\beta_{r_{*}}+\bar{\varepsilon}\right)>\mu_{\bar{N}}$, which is satisfied by definition of $\bar{N}$ in (5.2).

Third, for $1 \leq N \leq \bar{N}-1$, the calculations (4.36)-(4.38) are still valid with number $\widetilde{\delta}_{N}$ in (4.38) being changed to $\widetilde{\delta}_{N}=\lambda_{*}\left(\beta_{r_{*}}+\bar{\varepsilon}\right)-\mu_{N+1}$. Note that $\widetilde{\delta}_{N} \geq \lambda_{*}\left(\beta_{r_{*}}+\bar{\varepsilon}\right)-\mu_{\bar{N}}>0$.

We do finite induction in $N$ for $1 \leq N \leq \bar{N}$ and obtain $\left(\mathcal{T}_{\bar{N}}\right)$, which, by (4.32), yields (5.3). Here, each polynomial $q_{n}(t)$, for $1 \leq n \leq \bar{N}$, satisfies equation (4.20) with $\mathcal{J}_{n}$ being given by (4.21) particularly; that is, we obtain equation (5.4).

Next, we relax the regularity requirements for $F$ and $F_{k}$ 's.

Regarding $F$, its local Lipschitz property is imposed to guarantee the existence and uniqueness of solutions at least starting with small initial data. However, in some problems, $F$ is not that regular, but a small solution $y(t)$, for $t \in[0, \infty)$, already exists and is given. Then our results obtained above apply to this solution $y(t)$.

Regarding $F_{k}$ 's, what we need in the proofs of Theorems 4.3 and 5.1 is that each $F_{k}$, in addition to being positively homogeneous, has the Taylor series approximation of all orders about $\xi_{*}$, where $\xi_{*}$ is from Theorem 3.3. Because $\xi_{*}$ depends on $y(t)$ and varies in $\mathbb{R}_{0}^{d}$, function $F_{k}$ is required in Assumption 4.1 to be smooth on the entire set $\mathbb{R}_{0}^{d}$. However, in many cases, $F_{k}$ is only known to be smooth on an open set $V$ strictly smaller than $\mathbb{R}_{0}^{d}$. Then one needs $\xi_{*}$ to belong to $V$ as well. This is possible when more information about $\xi_{*}$, as an eigenvector of matrix $A$, is provided.

These two points will be reflected in Theorem 5.3 below.

Definition 5.2. For an open set $V$ in $\mathbb{R}^{d}$, denote by $\mathcal{X}(V)$, respectively $\mathcal{X}^{0}(V)$, the set of locally Lipschitz continuous, respectively continuous, functions on $\mathbb{R}^{d}$, with approximation (4.3) or (4.4) where $F_{k} \in \mathcal{H}_{\beta_{k}}\left(\mathbb{R}^{d}\right) \cap C^{\infty}(V)$ for all respective $k$ 's. The sets $\widehat{\mathcal{X}}(V)$ and $\widehat{\mathcal{X}}^{0}(V)$ are defined similarly with (5.1) replacing (4.3) and (4.4). In particular, denote $\mathcal{X}=\mathcal{X}\left(\mathbb{R}_{0}^{d}\right)$ and $\mathcal{X}^{0}=\mathcal{X}^{0}\left(\mathbb{R}_{0}^{d}\right)$. 
Note that $\mathcal{X}$ is the set of functions that satisfy Assumption 4.1 .

An extension of the results in Theorems 4.3 and 5.1 is the following general theorem.

Theorem 5.3. Suppose that all eigenvectors of matrix $A$ belong to an open set $V$ in $\mathbb{R}^{d}$.

(i) Then Theorem 4.3 applies to any function $F \in \mathcal{X}(V)$, and Theorem 5.1 applies to any function $F \in \widehat{\mathcal{X}}(V)$, for any non-trivial, decaying solution $y(t)$ of (1.4).

(ii) If $F \in \mathcal{X}^{0}(V)$, respectively $F \in \widehat{\mathcal{X}}^{0}(V)$, then Theorem 4.3, respectively Theorem 5.1, still holds true for a solution $y(t) \in C^{1}([0, \infty))$ of (1.4) that satisfies $y(t) \rightarrow 0$ as $t \rightarrow \infty$, and there is a divergent, strictly increasing sequence $\left(t_{n}\right)_{n=1}^{\infty}$ in $(0, \infty)$ such that $y\left(t_{n}\right) \neq 0$ for all $n \in \mathbb{N}$.

Proof. (i) In the proofs of Theorems 4.3 and [5.1, the eigenvector $\xi_{*}$ belongs to $V$, and, thanks to the condition $F_{k} \in C^{\infty}(V)$, we can still use the Taylor expansions of $F_{k}$ 's about $\xi_{*}$. Therefore, both proofs are unchanged and produce respective conclusions.

(ii) We re-examine Proposition 3.2. Select $T_{\varepsilon}=t_{n}$ for sufficiently large $n$ such that (3.9) still holds. Then we still obtain upper bound (3.5). With $y\left(T_{\varepsilon}\right)=y\left(t_{n}\right) \neq 0$, the estimate (3.13) holds for some $C_{2}^{\prime}>0$. Thus, the inequality in (3.6) holds for all $t \geq T_{\varepsilon}$. With such a lower bound of $|y(t)|$, we can still prove Theorem 3.3. After that, the argument in (i) continues to be valid.

The sets defined in Definition 5.2 and used in Theorem 5.3 will be explored more in section 6 below. Here, we state their very first property.

Proposition 5.4. For any open set $V$ in $\mathbb{R}^{d}$, the sets $\mathcal{X}(V), \widehat{\mathcal{X}}(V) \mathcal{X}^{0}(V)$ and $\widehat{\mathcal{X}}^{0}(V)$ are linear spaces.

Proof. We gives a proof for $\mathcal{X}(V)$, the other sets can be proved similarly. Thanks to Remark 4.4, it suffices to prove that the sum of any two functions of the form (4.3) is also of the form (4.3). Suppose $F(x)$ is the same as in (4.3), and

$$
G(x) \sim \sum_{k=1}^{\infty} G_{k}(x)
$$

where each $G_{k}$ is similar to $F_{k}$, but with degree $\beta_{k}^{\prime}>1$ instead of $\beta_{k}$. Arrange the set $\left\{\beta_{k}, \beta_{j}^{\prime}: k, j \in \mathbb{N}\right\}$ as an strictly increasing sequence $\left(\bar{\beta}_{k}\right)_{k=1}^{\infty}$. Clearly, $\bar{\beta}_{k} \rightarrow \infty$ as $k \rightarrow \infty$, and $\left(\beta_{k}\right)_{k=1}^{\infty}$ and $\left(\beta_{k}^{\prime}\right)_{k=1}^{\infty}$ are subsequences of $\left(\bar{\beta}_{k}\right)_{k=1}^{\infty}$. By inserting the zero function into (4.3) and (5.5) when needed, one can rewrite (and verify) $F$ and $G$ as

$$
F(x) \sim \sum_{k=1}^{\infty} \widetilde{F}_{k}(x) \text { and } G(x) \sim \sum_{k=1}^{\infty} \widetilde{G}_{k}(x),
$$

where $\widetilde{F}_{k}(x)$ and $\widetilde{G}_{k}(x)$ are in $C^{\infty}(V)$, positively homogeneous of the same degree $\bar{\beta}_{k}$. Then, $F+G$ is, obviously, of the form (4.3) with $\widetilde{F}_{k}+\widetilde{G}_{k}$ replacing $F_{k}$, and $\bar{\beta}_{k}$ replacing $\beta_{k}$.

\section{SPECIFIC CASES AND EXAMPLES}

We specify many cases for the function $F$ in Theorem 5.3, i.e., describe classes of functions in the spaces $\mathcal{X}(V), \widehat{\mathcal{X}}(V) \mathcal{X}^{0}(V)$ and $\widehat{\mathcal{X}}^{0}(V)$ in Definition 5.2 . 
For $n \in \mathbb{N}, p \in[1, \infty)$ and $x=\left(x_{1}, x_{2}, \ldots, x_{n}\right) \in \mathbb{R}^{n}$, the $\ell^{p}$-norm of $x$ is

$$
\|x\|_{p}=\left(\sum_{j=1}^{n}\left|x_{j}\right|^{p}\right)^{1 / p} \text {. }
$$

We recall that all these norms $\|\cdot\|_{p}$ on $\mathbb{R}^{n}$ are equivalent to each others.

For any $n \in \mathbb{N}, p \geq 1$ and $\alpha>0$, one has the following.

(a) The function $x \in \mathbb{R}^{n} \mapsto\|x\|_{p}^{\alpha}$ belongs to $C\left(\mathbb{R}^{n}\right) \cap C^{\infty}\left(\mathbb{R}_{*}^{n}\right) \cap \mathcal{H}_{\alpha}\left(\mathbb{R}^{n}\right)$.

(b) Assume, additionally, that $p$ is an even number. Then the function $x \in \mathbb{R}^{d} \mapsto\|x\|_{p}^{\alpha}$ belongs to $C^{\infty}\left(\mathbb{R}_{0}^{n}\right)$.

The first class of functions in $\mathcal{X}$ we describe is in the next theorem, which involves the $\ell^{p}$-norms of $x$ and polynomials on $\mathbb{R}^{d}$.

Theorem 6.1. Let $\delta>0$ and $m \in \mathbb{N}$. Suppose $G:(-\delta, \infty)^{m} \rightarrow \mathbb{R}$ be a $C^{\infty}$-function with $G(0)=0$, and $G_{0}: \mathbb{R}^{d} \rightarrow \mathbb{R}^{d}$ is a homogeneous polynomial of degree $m_{0} \in \mathbb{Z}_{+}$. Define a function $F: \mathbb{R}^{d} \mapsto \mathbb{R}^{d}$ by

$$
F(x)=G\left(\|x\|_{p_{1}}^{s_{1}},\|x\|_{p_{2}}^{s_{2}}, \ldots,\|x\|_{p_{m}}^{s_{m}}\right) G_{0}(x) \text { for } x \in \mathbb{R}^{d}
$$

where $p_{j} \in[1, \infty)$ and $s_{j} \in(0, \infty)$ for $j=1,2, \ldots, m$, are given real numbers.

Let $\bar{s}=\min \left\{s_{j}: j=1,2, \ldots, m\right\}$. Assume $\bar{s}+m_{0}>1$. Then the following statements hold true.

(i) $F(0)=0$ and $F \in C\left(\mathbb{R}^{d}\right) \cap C^{\infty}\left(\mathbb{R}_{*}^{d}\right)$.

(ii) $F \in \mathcal{X}^{0}\left(\mathbb{R}_{*}^{d}\right)$.

(iii) If $p_{1}, \ldots, p_{m}>1$, then $F \in C^{1}\left(\mathbb{R}^{d}\right)$, and, consequently, $F$ is locally Lipschitz in $\mathbb{R}^{d}$.

(iv) If $p_{1}, p_{2}, \ldots, p_{m}$ are even numbers, then $F \in \mathcal{X}$.

Proof. In part (i), the property $F(0)=0$ follows the fact $G(0)=0$. The proof of the remaining statement in (i) is elementary, using the chain rule for derivatives and property (a) right before this theorem.

We prove (ii). By using the Taylor expansion of $G(z)$, for $z \in(-\delta, \infty)^{m}$, about the origin of $\mathbb{R}^{m}$, we can approximate $G\left(\|x\|_{p_{1}}^{s_{1}},\|x\|_{p_{2}}^{s_{2}}, \ldots,\|x\|_{p_{m}}^{s_{m}}\right)$, for $k \in \mathbb{N}$, by

$$
\sum_{\substack{\gamma=\left(\gamma_{1}, \gamma_{2}, \ldots, \gamma_{m}\right) \in \mathbb{Z}_{+}^{m} \\|\gamma| \leq k}} c_{\gamma}\|x\|_{p_{1}}^{s_{1} \gamma_{1}}\|x\|_{p_{2}}^{s_{2} \gamma_{2}} \ldots\|x\|_{p_{m}}^{s_{m} \gamma_{m}}
$$

with the remainder being

$$
\mathcal{O}\left(\left(\|x\|_{p_{1}}^{s_{1}}+\|x\|_{p_{2}}^{s_{2}}+\ldots+\|x\|_{p_{m}}^{s_{m}}\right)^{k+1}\right)=\mathcal{O}\left(|x|^{\bar{s}(k+1)}\right) \text { as } x \rightarrow 0
$$

where each $\gamma$ is a multi-index with length

$$
|\gamma|=\gamma_{1}+\gamma_{2}+\ldots+\gamma_{m}, \text { and } c_{\gamma}=\frac{1}{\gamma_{1} ! \gamma_{2} ! \ldots \gamma_{m} !} \cdot \frac{\partial^{|\gamma|} G(0)}{\partial x_{1}^{\gamma_{1}} \partial x_{2}^{\gamma_{2}} \ldots \partial x_{m}^{\gamma_{m}}} .
$$

Re-arrange the set

$$
\left\{m_{0}+\sum_{j=1}^{m} s_{j} \gamma_{j}: \gamma_{j} \in \mathbb{Z}_{+},\left(\gamma_{1}, \gamma_{2}, \ldots, \gamma_{m}\right) \neq 0\right\}
$$

as a strictly increasing sequence $\left(\beta_{k}\right)_{k=1}^{\infty}$. Note that $\beta_{k} \rightarrow \infty$ as $k \rightarrow \infty$, and, because of the assumption $\bar{s}+m_{0}>1$, we have $\beta_{k}>1$ for all $k \in \mathbb{N}$. 
Then we can re-write $F(x)$ in the form of (4.3), where

$$
F_{k}(x)=\sum_{\substack{\gamma=\left(\gamma_{1}, \gamma_{2}, \ldots, \gamma_{m}\right) \in \mathbb{Z}_{+}^{m}, m_{0}+\sum_{j=1}^{m} s_{j} \gamma_{j}=\beta_{k}}} c_{\gamma}\|x\|_{p_{1}}^{s_{1} \gamma_{1}}\|x\|_{p_{2}}^{s_{2} \gamma_{2}} \ldots\|x\|_{p_{m}}^{s_{m} \gamma_{m}} G_{0}(x)
$$

By property (a) right before this theorem and property $(\mathrm{d})$ after Definition $2.2, F_{k} \in$ $\mathcal{H}_{\beta_{k}}\left(\mathbb{R}^{d}\right) \cap C^{\infty}\left(\overline{\mathbb{R}_{*}^{d}}\right)$. By this and the facts $F(0)=0, F \in C\left(\mathbb{R}^{d}\right)$ in $(\mathrm{i})$, we conclude $F \in$ $\mathcal{X}^{0}\left(\mathbb{R}_{*}^{d}\right)$.

We prove (iii). Because $G_{0}$ is a homogeneous polynomial of degree $m_{0}$, there is $C>0$ such that $G_{0}(x)$ and its derivative matrix $D G_{0}(x)$ can be estimated, for any $x \in \mathbb{R}^{d}$, by

$$
\left|G_{0}(x)\right| \leq C|x|^{m_{0}} \text { and }\left|D G_{0}(x)\right| \begin{cases}\leq C|x|^{m_{0}-1} & \text { if } m_{0} \geq 1 \\ =0 & \text { if } m_{0}=0\end{cases}
$$

By using the linear approximation of $G(z)$ for $z$ near 0 in $\mathbb{R}^{m}$, we have

$$
G(z)=\mathcal{O}(|z|)=\mathcal{O}\left(\left|z_{1}\right|+\ldots+\left|z_{m}\right|\right), \text { as } z=\left(z_{1}, \ldots, z_{m}\right) \rightarrow 0 .
$$

Applying this property to $z=\left(\|x\|_{p_{1}}^{s_{1}},\|x\|_{p_{2}}^{s_{2}}, \ldots,\|x\|_{p_{m}}^{s_{m}}\right)$, we have

$$
G\left(\|x\|_{p_{1}}^{s_{1}},\|x\|_{p_{2}}^{s_{2}}, \ldots,\|x\|_{p_{m}}^{s_{m}}\right)=\mathcal{O}\left(\|x\|_{p_{1}}^{s_{1}}+\|x\|_{p_{2}}^{s_{2}}+\ldots+\|x\|_{p_{m}}^{s_{m}}\right)=\mathcal{O}\left(|x|^{\bar{s}}\right) \text { as } x \rightarrow 0
$$

and, together with the first inequality in (6.4),

$$
F(x)=\mathcal{O}\left(|x|^{\bar{s}+m_{0}}\right) \text { as } x \rightarrow 0 .
$$

Since $\bar{s}+m_{0}>1$ and $F(0)=0$, it follows (6.5) that

$$
D F(0)=0 .
$$

For $1 \leq i \leq m$ and $1 \leq j \leq d$, one has the partial derivative, thanks to $p_{i}>1$,

$$
x=\left(x_{1}, \ldots, x_{d}\right) \in \mathbb{R}^{d} \mapsto \frac{\partial\left(\left|x_{j}\right|^{p_{i}}\right)}{\partial x_{j}}=p_{i}\left|x_{j}\right|^{p_{i}-1} \operatorname{sign}\left(x_{j}\right),
$$

which is a continuous function on $\mathbb{R}^{d}$.

For $x \in \mathbb{R}^{d} \backslash\{0\}$ and $j=1,2, \ldots, d$, we have

$$
\begin{aligned}
\frac{\partial F(x)}{\partial x_{j}}= & \left.\sum_{i=1}^{m} \frac{\partial G(z)}{\partial z_{i}}\right|_{z=\left(\|x\|_{p_{1}}^{s_{1}},\|x\|_{p_{2}}^{s_{2}}, \ldots,\|x\|_{p_{m}}^{s_{m}}\right)} s_{i}\|x\|_{p_{i}}^{s_{i}-p_{i}}\left|x_{j}\right|^{p_{i}-1} \operatorname{sign}\left(x_{j}\right) G_{0}(x) \\
& +G\left(\|x\|_{p_{1}}^{s_{1}},\|x\|_{p_{2}}^{s_{2}}, \ldots,\|x\|_{p_{m}}^{s_{m}}\right) \frac{\partial G_{0}(x)}{\partial x_{j}} .
\end{aligned}
$$

Clearly, $\partial F(x) / \partial x_{j}$ is continuous on $\mathbb{R}^{d} \backslash\{0\}$. Consider its continuity at the origin. For the first summation on the right-hand side of (6.7),

$$
\left.\frac{\partial G(z)}{\partial z_{i}}\right|_{z=\left(\|x\|_{p_{1}}^{s_{1}},\|x\|_{p_{2}, \ldots,}^{s_{2}},\|x\|_{p_{m}}^{s_{m}}\right)}=\mathcal{O}(1) \text { as } x \rightarrow 0,
$$

and, thanks to the first estimate in (6.4),

$$
\|x\|_{p_{i}}^{s_{i}-p_{i}}\left|x_{j}\right|^{p_{i}-1}\left|\operatorname{sign}\left(x_{j}\right) G_{0}(x)\right| \leq \mathcal{O}\left(|x|^{s_{i}-1}|x|^{m_{0}}\right)=\mathcal{O}\left(|x|^{\bar{s}+m_{0}-1}\right) \text { as } x \rightarrow 0 .
$$


By the second estimate in (6.4), the last term in (6.7), it is zero when $m=0$, and can be estimated, when $m_{0} \geq 1$, by

$$
\left|G\left(\|x\|_{p_{1}}^{s_{1}},\|x\|_{p_{2}}^{s_{2}}, \ldots,\|x\|_{p_{m}}^{s_{m}}\right) \frac{\partial G_{0}(x)}{\partial x_{j}}\right| \leq \mathcal{O}\left(|x|^{\bar{s}}\right) C|x|^{m_{0}-1}=\mathcal{O}\left(|x|^{\bar{s}+m_{0}-1}\right) \text { as } x \rightarrow 0 .
$$

The above estimates from (6.8) to (6.9) for the right-hand side of (6.7) yield

$$
\lim _{x \rightarrow 0} \frac{\partial F(x)}{\partial x_{j}}=0 .
$$

Together with (6.6), this limit implies that $\partial F(x) / \partial x_{j}$ is continuous at the origin for $j=$ $1,2, \ldots, d$. Therefore, $F \in C^{1}\left(\mathbb{R}^{d}\right)$, and, consequently, $F$ is locally Lipschitz in $\mathbb{R}^{d}$.

Finally, we prove (iv). In case all $p_{j}$ 's are even numbers, then, by property (b) right before Theorem 6.1, all $F_{k}$ 's in $(\underline{6.3})$ belong to $C^{\infty}\left(\mathbb{R}_{0}^{d}\right)$. Combining this fact with (ii) and (iii) above, we have $F \in \mathcal{X}$.

Example 6.2. Let $\alpha$ be any number in $(0, \infty)$ that is not an even integer, and

$$
F(x)=|x|^{\alpha} x \text { for } x \in \mathbb{R}^{d} .
$$

Applying Theorem 6.1(iv) to $m=1, G(z)=z$ for $z \in \mathbb{R}, G_{0}(x)=x, p_{1}=2$ and $s_{1}=\alpha$, we have $F \in \mathcal{X}$. Even in this simple case, the asymptotic expansions obtained in Theorem 4.3 is new.

Example 6.3. Given a constant $d \times d$ matrix $M_{0}$, even numbers $p_{1}, p_{2} \geq 2$, and real numbers $\alpha, \beta>0$, let

$$
F(x)=\frac{\|x\|_{p_{1}}^{\alpha} M_{0} x}{1+\|x\|_{p_{2}}^{\beta}} \text { for } x \in \mathbb{R}^{d} .
$$

Applying Theorem 6.1](iv) to functions $G\left(z_{1}, z_{2}\right)=z_{1} /\left(1+z_{2}\right), G_{0}(x)=M_{0} x$ and numbers $s_{1}=\alpha, s_{2}=\beta$, one has $F \in \mathcal{X}$. The explicit form of (4.3) can be obtained quickly as follows.

For $x \in \mathbb{R}^{d}$ with $\|x\|_{p_{2}}<1$, we expand $1 /\left(1+\|x\|_{p_{2}}^{\beta}\right)$, using the geometric series, and can verify that

$$
F(x) \sim \sum_{k=1}^{\infty}(-1)^{k-1}\|x\|_{p_{1}}^{\alpha}\|x\|_{p_{2}}^{(k-1) \beta} M_{0} x
$$

in the sense of (H1) in Assumption 4.1. This yields (4.3) with $\beta_{k}=1+\alpha+(k-1) \beta$.

When $\|\cdot\|_{p_{1}}=\|\cdot\|_{p_{2}}=|\cdot|$, function $F$ in (6.11) covers the particular case discussed in (1.5), and expansion (6.12) simply reads as

$$
F(x) \sim \sum_{k=1}^{\infty}(-1)^{k-1}|x|^{\alpha+(k-1) \beta} M_{0} x .
$$

Example 6.4. For $k \in \mathbb{N}$, let $M_{k}$ be a constant $d \times d$ matrix, and $p_{k} \geq 2$ be an even number, and $\alpha_{k}>0$.

(a) Each function $x \in \mathbb{R}^{d} \mapsto\|x\|_{p_{k}}^{\alpha_{k}} M_{k} x$ can play the role of $F_{k}$ in (4.3) or (5.1). In this case, we write, respectively,

$$
F(x) \sim \sum_{k=1}^{\infty}\|x\|_{p_{k}}^{\alpha_{k}} M_{k} x, \text { or }\left|F(x)-\sum_{k=1}^{N_{*}}\|x\|_{p_{k}}^{\alpha_{k}} M_{k} x\right|=\mathcal{O}\left(|x|^{\alpha_{N_{*}}+1+\bar{\varepsilon}}\right) \text { as } x \rightarrow 0 .
$$


In particular, thanks to Theorem 6.1](iv)], the function

$$
F(x)=\sum_{k=1}^{N_{*}}\|x\|_{p_{k}}^{\alpha_{k}} M_{k} x \text {, for } x \in \mathbb{R}^{d} \text {, belongs to } \mathcal{X} \text {. }
$$

(b) We can replace $M_{k} x$ in (6.13) with an $\mathbb{R}^{d}$-valued homogeneous polynomial in $x$ of degree $m_{k} \in \mathbb{Z}_{+}$. Of course, the set $\left\{\alpha_{k}+m_{k}: k \in \mathbb{N}\right\}$ is required to be in $(1, \infty)$ and can be re-arranged as a sequence that strictly increases to infinity.

In Examples 6.2, 6.3 and 6.4 above, we can also consider more complicated variations. For example, in (6.10), (6.11) and (6.13), we can replace $|x|$ or $\|x\|_{p_{k}}$ with $\left\|S_{k} x\right\|_{p_{k}}$, where $S_{k}$ 's are invertible $d \times d$ matrices.

Note that a positively homogeneous function of the form (2.6), in general, does not belong to $C^{\infty}\left(\mathbb{R}_{0}^{d}\right)$. Hence, it cannot play a role of an $F_{k}$ in (4.3) or (5.1). However, in some cases, see (6.14) and (6.15) below, it can.

Theorem 6.5. Consider function $F(x)$ given by (2.6) with $X=Y=\mathbb{R}^{d}, s \geq 1$ and $\left(Y_{j},\|\cdot\|_{Y_{j}}\right)=\left(\mathbb{R}^{n_{j}},\|\cdot\|_{p_{j}}\right)$ for $j=1, \ldots, s$. Suppose, for $j=1, \ldots, s$,

the number $p_{j}$ is even, and

the only solution of equation $P_{j}(x)=0$ is $x=0$.

(i) One has $F \in \mathcal{H}_{\beta}\left(\mathbb{R}^{d}\right) \cap C\left(\mathbb{R}^{d}\right) \cap C^{\infty}\left(\mathbb{R}_{0}^{d}\right)$, where number $\beta$ is defined in (2.7).

(ii) If $\beta>1$, then $F \in \mathcal{X}^{0}$.

(iii) Let $\bar{\nu}=\min \left\{\nu_{j}: j=1, \ldots, s\right\}$ and assume $m_{0}+\bar{\nu}>1$. Then $F \in C^{1}\left(\mathbb{R}^{d}\right)$. Consequently, $F \in \mathcal{X}$.

Proof. For part (i), the fact $F \in \mathcal{H}_{\beta}\left(\mathbb{R}^{d}\right)$ is due to (2.7), while the other fact $F \in C\left(\mathbb{R}^{d}\right) \cap$ $C^{\infty}\left(\mathbb{R}_{0}^{d}\right)$ is clear. Part (ii) comes from part (i).

We prove part (iii) now. Same as (6.4), there is $C>0$ such that, for $j=0,1, \ldots, s$, and any $x \in \mathbb{R}^{d}$,

$$
\left|P_{j}(x)\right| \leq C|x|^{m_{j}} \text { and }\left|D P_{j}(x)\right| \begin{cases}\leq C|x|^{m_{j}-1} & \text { if } m_{j} \geq 1, \\ =0 & \text { if } m_{j}=0,\end{cases}
$$

Because $s \geq 1$ and $m_{j} \geq 1$ for $j \geq 1$, we have $\beta=m_{0}+\sum_{j=1}^{s} m_{j} \nu_{j} \geq m_{0}+\bar{\nu}>1$.

Note that $F(0)=0$ and, by the first estimate in (6.16),

$$
F(x)=\mathcal{O}\left(|x|^{m_{0}+\sum_{j=1}^{m} \nu_{j} m_{j}}\right)=\mathcal{O}\left(|x|^{\beta}\right) \text { as } x \rightarrow 0 .
$$

Then, thanks to $\beta>1$, we have the derivative matrix $D F(0)=0$.

For $j=1,2, \ldots, s$, write $P_{j}=\left(P_{j, 1}, P_{j, 2}, \ldots, P_{j, n_{j}}\right)$.

Let $x=\left(x_{1}, \ldots, x_{d}\right) \in \mathbb{R}^{d} \backslash\{0\}$. Then, thanks to condition (6.15), $P_{j}(x) \neq 0$ for $j=$ $1,2, \ldots, s$. For $i=1,2, \ldots, d$, we have the partial derivative

$$
\begin{aligned}
& \frac{\partial F(x)}{\partial x_{i}}=\left\|P_{1}(x)\right\|_{p_{1}}^{\nu_{1}}\left\|P_{2}(x)\right\|_{p_{2}}^{\nu_{2}} \ldots\left\|P_{s}(x)\right\|_{p_{s}}^{\nu_{s}} \frac{\partial P_{0}(x)}{\partial x_{i}} \\
& +\left\{\sum_{j=1}^{s}\left(\prod_{\substack{1 \leq j^{\prime} \leq s, j^{\prime} \neq j}}\left\|P_{j^{\prime}}(x)\right\|_{p_{j^{\prime}}}^{\nu_{j^{\prime}}}\right) \nu_{j}\left\|P_{j}(x)\right\|_{p_{j}}^{\nu_{j}-p_{j}}\left(\sum_{\ell=1}^{n_{j}}\left(P_{j, \ell}(x)\right)^{p_{j}-1} \frac{\partial P_{j, \ell}(x)}{\partial x_{i}}\right)\right\} P_{0}(x) .
\end{aligned}
$$


One can see that this partial derivative is continuous on $\mathbb{R}^{d} \backslash\{0\}$. For the continuity of $\partial F(x) / \partial x_{j}$ at the origin, we estimate the right-hand side of (6.17). On the one hand,

$$
\left\|P_{1}(x)\right\|_{p_{1}}^{\nu_{1}}\left\|P_{2}(x)\right\|_{p_{2}}^{\nu_{2}} \ldots\left\|P_{s}(x)\right\|_{p_{s}}^{\nu_{s}}\left|\frac{\partial P_{0}(x)}{\partial x_{j}}\right| \text { is zero if } m_{0}=0,
$$

or, in the case $m_{0} \geq 1$, it can be estimated, with the use of (6.16), by

$$
\left\|P_{1}(x)\right\|_{p_{1}}^{\nu_{1}}\left\|P_{2}(x)\right\|_{p_{2}}^{\nu_{2}} \ldots\left\|P_{s}(x)\right\|_{p_{s}}^{\nu_{s}}\left|\frac{\partial P_{0}(x)}{\partial x_{j}}\right| \leq C^{\prime}|x|^{\sum_{j=1}^{s} m_{j} \nu_{j}}|x|^{m_{0}-1}=C^{\prime}|x|^{\beta-1},
$$

for some generic constant $C^{\prime}>0$. Here, and also in calculations below, we use the equivalence between any norm $\|\cdot\|_{p_{j}}$ and $|\cdot|$.

On the other hand, for each $j=1, \ldots, s$, and $\ell=1, \ldots, n_{j}$, by using the estimates in (6.16) again, we have

$$
\begin{aligned}
& \nu_{j}\left(\prod_{\substack{1 \leq j^{\prime} \leq s, j^{\prime} \neq j}}\left\|P_{j^{\prime}}(x)\right\|_{p_{j^{\prime}}}^{\nu_{j^{\prime}}}\right)\left\|P_{j}(x)\right\|_{p_{j}}^{\nu_{j}-p_{j}}\left|P_{j, \ell}(x)\right|^{p_{j}-1}\left|\frac{\partial P_{j, \ell}(x)}{\partial x_{i}}\right|\left|P_{0}(x)\right| \\
& \leq C^{\prime}\left(\prod_{\substack{1 \leq j^{\prime} \leq s, j^{\prime} \neq j}}|x|^{m_{j^{\prime}} \nu_{j^{\prime}}}\right)\left\|P_{j}(x)\right\|_{p_{j}}^{\nu_{j}-1}|x|^{m_{j}-1}|x|^{m_{0}} \\
& \leq C^{\prime}\left(\prod_{\substack{1 \leq j^{\prime} \leq m_{j}, j^{\prime} \neq j}}|x|^{m_{j^{\prime}} \nu_{j^{\prime}}}\right)|x|^{m_{j}\left(\nu_{j}-1\right)}|x|^{m_{j}-1}|x|^{m_{0}}=C^{\prime}|x|^{m_{0}+\sum_{j^{\prime}=1}^{m} \nu_{j^{\prime}} m_{j^{\prime}}-1}=C^{\prime}|x|^{\beta-1} .
\end{aligned}
$$

Summing up the above estimates after (6.17) and passing $x \rightarrow 0$, with $\beta>1$, give

$$
\lim _{x \rightarrow 0} \frac{\partial F(x)}{\partial x_{i}}=0=\frac{\partial F(0)}{\partial x_{i}} .
$$

The last relation comes from the fact $D F(0)=0$ obtained earlier. Thus, $\partial F / \partial x_{i}$ is continuous on $\mathbb{R}^{d}$, for $i=1, \ldots, d$. Because $F \in C\left(\mathbb{R}^{d}\right)$ from part (i), we obtain $F \in C^{1}\left(\mathbb{R}^{d}\right)$. Consequently, $F$ is locally Lipschitz, and, by combining this with the facts in part (i), we conclude $F \in \mathcal{X}$.

In Theorem 6.5, we usually consider the case $\nu_{j} / p_{j} \notin \mathbb{N}$ for all $j$. Indeed, for an index $j$ with $\nu_{j} / p_{j} \in \mathbb{N}$, the corresponding term $\left\|P_{j}(x)\right\|_{p_{j}}^{\nu_{j}}$ is a polynomial, and we can combine it with the polynomial $P_{0}(x)$.

Example 6.6. Regarding condition (6.15), it can be met for many forms of $P_{j}$. For example, if $P_{j}(x)=\left(x^{T} M_{1} x\right) M_{0} x$ for $x \in \mathbb{R}^{d}$, where $M_{1}$ is a positive definite $d \times d$ matrix, and $M_{0}$ is an invertible $d \times d$ matrix, then $P_{j}$ satisfies (6.15).

Example 6.7. Consider $d=2$ and let

$$
F\left(x_{1}, x_{2}\right)=\left(\left|x_{1}^{3}-x_{2}^{3}\right|^{p_{1}}+\left|x_{1}^{3}+x_{2}^{3}\right|^{p_{1}}\right)^{\alpha / p_{1}} \cdot\left(\left|x_{1} x_{2}\right|^{p_{2}}+\left|3 x_{1}^{2}-2 x_{2}^{2}\right|^{p_{2}}\right)^{\beta / p_{2}} M_{0}\left(x_{1}, x_{2}\right),
$$


where $p_{1}, p_{2} \geq 2$ are even numbers, $M_{0}$ is a $\mathbb{R}^{2}$-valued homogeneous polynomials of degree $m_{0} \in \mathbb{Z}_{+}$, and $\alpha, \beta>0$. Then $F$ is of the form (2.6) with $s=2, n_{1}=n_{2}=2, m_{1}=3$, $\nu_{1}=\alpha, m_{2}=2, \nu_{2}=\beta$, and

$$
P_{1}(x)=\left(x_{1}^{3}-x_{2}^{3}, x_{1}^{3}+x_{2}^{3}\right), \quad P_{2}(x)=\left(x_{1} x_{2}, 3 x_{1}^{2}-2 x_{2}^{2}\right) .
$$

One can verify that $P_{1}$ and $P_{2}$ satisfy (6.15). If $m_{0}+\min \{\alpha, \beta\}>1$, then, thanks to Theorem 6.5) $F \in \mathcal{X}$.

In the remainder of this section, we focus on functions constituted essentially by $x_{i}^{\gamma_{i}}$, where $x_{i}$ 's are coordinates of a vector $x \in \mathbb{R}^{d}$. We will consider more general forms of these power functions, and also combine them with other positively homogeneous functions such as $\|x\|_{p_{i}}^{\gamma_{i}}$.

Notation 6.8. We will use the following notation for different types of power functions.

- Define $\omega$, a subset of $\mathbb{R}^{2}$, by $\omega=\left(\mathbb{Z}_{+} \times\{0\}\right) \cup([0, \infty) \times\{-1,1\})$.

- For $x \in \mathbb{R}$ and $(\gamma, \tau) \in \omega$, denote $\langle x\rangle_{\tau}^{\gamma}$ as follows

$$
\begin{aligned}
& \langle x\rangle_{0}^{0}=\langle x\rangle_{1}^{0}=\langle x\rangle_{-1}^{0}=1, \text { for } \gamma=0, \text { and } \\
& \langle x\rangle_{0}^{\gamma}=x^{\gamma}, \quad\langle x\rangle_{1}^{\gamma}=|x|^{\gamma}, \quad\langle x\rangle_{-1}^{\gamma}=|x|^{\gamma} \operatorname{sign}(x), \text { for } \gamma>0 .
\end{aligned}
$$

- For $\gamma=\left(\gamma_{1}, \gamma_{2}, \ldots, \gamma_{n}\right) \in \mathbb{R}^{n}$ and $\tau=\left(\tau_{1}, \tau_{2}, \ldots, \tau_{n}\right) \in \mathbb{R}^{n}$, denote

$$
[\tau, \gamma]=\left(\left(\gamma_{1}, \tau_{1}\right),\left(\gamma_{2}, \tau_{2}\right), \ldots,\left(\gamma_{n}, \tau_{n}\right)\right) \in\left(\mathbb{R}^{2}\right)^{n}
$$

- For vector $x=\left(x_{1}, x_{2}, \ldots, x_{n}\right) \in \mathbb{R}^{n}$, multi-index $\gamma=\left(\gamma_{1}, \gamma_{2}, \ldots, \gamma_{n}\right) \in[0, \infty)^{n}$ and $\tau=\left(\tau_{1}, \tau_{2}, \ldots, \tau_{n}\right) \in\{-1,0,1\}^{n}$ with $[\gamma, \tau] \in \omega^{n}$, denote

$$
\langle x\rangle_{\tau}^{\gamma}=\left\langle x_{1}\right\rangle_{\tau_{1}}^{\gamma_{1}} \cdot\left\langle x_{2}\right\rangle_{\tau_{2}}^{\gamma_{2}} \ldots\left\langle x_{n}\right\rangle_{\tau_{n}}^{\gamma_{n}}
$$

- For $x \in \mathbb{R}^{n}, p=\left(p_{1}, p_{2}, \ldots, p_{n}\right) \in[1, \infty)^{n}$ and $\gamma=\left(\gamma_{1}, \gamma_{2}, \ldots, \gamma_{n}\right) \in[0, \infty)^{n}$, denote

$$
\|x\|_{p}^{\gamma}=\|x\|_{p_{1}}^{\gamma_{1}} \cdot\|x\|_{p_{2}}^{\gamma_{2}} \ldots\|x\|_{p_{n}}^{\gamma_{n}}
$$

with the convention $\|x\|_{p_{i}}^{0}=1$.

The last type of power in (6.19) can be used to re-write the terms like $\left|x_{i}\right|^{\alpha} x_{i}$ as $\left\langle x_{i}\right\rangle_{-1}^{\alpha+1}$. Also, when some power $\gamma_{i}$ in (6.20) is zero, then, thanks to (6.18), the corresponding term $\left\langle x_{i}\right\rangle_{\tau_{i}}^{\gamma_{i}}$ is 1 regardless the value of $x_{i}$.

Let $m \in \mathbb{N}, p \in[1, \infty)^{m}, \nu \in[0, \infty)^{m}$, and $\gamma, \tau \in \mathbb{R}^{d}$ with $[\gamma, \tau] \in \omega^{d}$, and a constant vector $c \in \mathbb{R}^{d}$. Then

the function $x \in \mathbb{R}^{d} \mapsto\|x\|_{p}^{\nu}\langle x\rangle_{\tau}^{\gamma} c$ belongs to $\mathcal{H}_{|\nu|+|\gamma|}\left(\mathbb{R}^{d}\right) \cap C\left(\mathbb{R}^{d}\right) \cap C^{\infty}\left(\mathbb{R}_{*}^{d}\right)$,

where $|\nu|$ and $|\gamma|$ denote the lengths of multi-indices, see (6.2).

In the following presentation, condition $|\nu|=0$ is used to indicate that the term $\|x\|_{p}^{\nu}$ is not present in (6.21). In this case, the values of $m$ and $p$ are irrelevant.

When, in general, the term $\langle x\rangle_{\tau}^{\gamma}$ is a homogeneous polynomial, or, in particular, $|\gamma|=0$, the function in (6.21) is reduced to the form (6.1), which was already dealt with in Theorem 6.1 .

Theorem 6.9. Assume that all eigenvectors of matrix $A$ belong to $V=\mathbb{R}_{*}^{d}$. 
(i) Suppose function $F: \mathbb{R}^{d} \rightarrow \mathbb{R}^{d}$ and number $\beta \in(1, \infty)$ satisfy that $F$ is a finite sum of the functions in (6.21) with $|\nu|+|\gamma|=\beta$. Then

$$
F(0)=0 \text { and } F \in \mathcal{H}_{\beta}\left(\mathbb{R}^{d}\right) \cap C\left(\mathbb{R}^{d}\right) \cap C^{\infty}(V) .
$$

Consequently, $F$ belongs to $\mathcal{X}^{0}(V)$, and can also play the role of a function $F_{k}$ in (4.3) or (5.1) with $\beta_{k}=\beta$.

(ii) Suppose $F$ is a finite sum of functions in (6.21) with multi-indices $\nu=\left(\nu_{1}, \ldots, \nu_{m}\right)$ and $\gamma=\left(\gamma_{1}, \ldots, \gamma_{d}\right)$ satisfying

(a) $|\nu|+|\gamma|>1$, and

(b) $|\nu|=0$ or $\left(\forall i=1, \ldots, m: \nu_{i} \geq 1\right)$, and

(c) $\forall j=1, \ldots, d: \gamma_{j}=0$ or $\gamma_{j} \geq 1$.

Then $F \in \mathcal{X}(V)$.

Proof. Part (i) clearly comes from property (6.21) and the fact $\beta>1$.

Consider part (ii). Thanks to Proposition 5.4, it suffices to prove (ii) for $F(x)=\|x\|_{p}^{\nu}\langle x\rangle_{\tau}^{\gamma} c$ given as in (6.21) with $p=\left(p_{1}, \ldots, p_{m}\right)$ and $\tau=\left(\tau_{1}, \ldots, \tau_{d}\right)$. By (6.21),$F \in \mathcal{H}_{\beta}\left(\mathbb{R}^{d}\right) \cap C^{\infty}(V)$, with $\beta=|\nu|+|\gamma|$, which is greater than 1 , thanks to condition (a). Conditions (b) and (c) guarantee that the functions $x \in \mathbb{R}^{d} \mapsto\|x\|_{p_{i}}^{\nu_{i}}$, for $i=1, \ldots, m$, and $x=\left(x_{1}, \ldots, x_{d}\right) \in$ $\mathbb{R}^{d} \mapsto\left\langle x_{j}\right\rangle_{\tau_{j}}^{\gamma_{j}}$, for $j=1, \ldots, d$, are locally Lipschitz on $\mathbb{R}^{d}$. Therefore, the function $F$, as a multiplication of these functions and the constant vector $c$, is locally Lipschitz. All together, we have $F \in \mathcal{X}(V)$.

Example 6.10. Consider the following system of ODEs in $\mathbb{R}^{2}$ :

$$
\begin{aligned}
& y_{1}^{\prime}+2 y_{1}+y_{2}=|y|^{2 / 3}\left|y_{1}\right|^{1 / 2} y_{2}^{3}, \\
& y_{2}^{\prime}+y_{1}+2 y_{2}=\|y\|_{5 / 2}^{1 / 3} y_{1}\left|y_{2}\right|^{1 / 4} \operatorname{sign}\left(y_{2}\right) .
\end{aligned}
$$

The corresponding matrix $A$ has eigenvalues and bases of the corresponding eigenspaces as follows: $\lambda_{1}=1$, basis $\{(-1,1)\}$, and $\lambda_{2}=3$, basis $\{(1,1)\}$. Then any eigenvector of $A$ belongs to $V=\mathbb{R}_{*}^{2}$. The corresponding function $F$ belongs to $\mathcal{X}^{0}(V)$, thanks to Theorem 6.9(i), and we can apply Theorem 5.3(ii).

Example 6.11. Consider the following system in $\mathbb{R}^{2}$ :

$$
\begin{aligned}
y_{1}^{\prime}+y_{1} & =-\left|y_{2}\right|^{\alpha} y_{1}, \\
y_{2}^{\prime}+y_{1}+2 y_{2} & =-y_{1}^{2} y_{2},
\end{aligned}
$$

where $\alpha>0$ is not an even integer. The matrix $A$, its eigenvalues and bases of corresponding eigenspaces are

$$
A=\left(\begin{array}{ll}
1 & 0 \\
1 & 2
\end{array}\right), \quad \begin{aligned}
& \lambda_{1}=1, \text { basis }\{(1,-1)\}, \\
& \lambda_{2}=2, \text { basis }\{(0,1)\} .
\end{aligned}
$$

In this case, $F=f+g$, where

$$
f\left(x_{1}, x_{2}\right)=\left(-\left|x_{2}\right|^{\alpha} x_{1}, 0\right) \in \mathcal{H}_{1+\alpha}\left(\mathbb{R}^{2}\right) \text { and } g\left(x_{1}, x_{2}\right)=\left(0,-x_{1}^{2} x_{2}\right) \in \mathcal{H}_{3}\left(\mathbb{R}^{2}\right) .
$$

One finds that any eigenvector of $A$ belongs to $V=\mathbb{R} \times \mathbb{R}_{*}$, and

$$
f, g \in C^{\infty}(V) \text {. }
$$

Hence, $F \in \mathcal{X}^{0}(V)$ and we can apply Theorem 5.3(ii).

In case $\alpha \geq 1$, we have $F$ is locally Lipschitz on $\mathbb{R}^{2}$. This fact, together with (6.23) and (6.24), implies that $F \in \mathcal{X}(V)$ and we can apply Theorem 5.3)(i). 
Example 6.12. There are many other situations, especially in multi-dimensional spaces higher than $\mathbb{R}^{2}$. We present one example here. Let $d=3$, and assume $3 \times 3$ matrix $A$ has the following eigenvalues and bases of the corresponding eigenspaces

$$
\lambda_{1}=\lambda_{2}=1 \text {, basis }\left\{\xi_{1}=(1,0,1), \xi_{2}=(0,1,0)\right\} \text {, and } \lambda_{3}=2 \text {, basis }\left\{\xi_{3}=(1,1,-1)\right\} .
$$

Let $F(x)=\left(x_{1}^{2}+x_{2}^{2}\right)^{1 / 3} \cdot\left(x_{2}^{6}+x_{3}^{6}\right)^{1 / 5} P(x)$, where $P$ is a polynomial vector field on $\mathbb{R}^{3}$ of degree $m_{0} \in \mathbb{N}$ without the constant term, i.e., $P(0)=0$.

Suppose $\xi$ is an eigenvector of $A$. Then $\xi=c_{1} \xi_{1}+c_{2} \xi_{2}$ for $c_{1}^{2}+c_{2}^{2}>0$, or $\xi=c_{3} \xi_{3}$ for $c_{3} \neq 0$. One can verify that

$$
\begin{aligned}
\xi \in V & =\left\{\left(x_{1}, x_{2}, x_{3}\right): x_{2} \neq 0 \text { or } x_{1} x_{3} \neq 0\right\} \\
& =\left(\mathbb{R} \times \mathbb{R}_{*} \times \mathbb{R}\right) \cup\left(\mathbb{R}_{*} \times \mathbb{R} \times \mathbb{R}_{*}\right)=\left(\mathbb{R}_{0}^{2} \times \mathbb{R}\right) \cap\left(\mathbb{R} \times \mathbb{R}_{0}^{2}\right) .
\end{aligned}
$$

Note that $F \in \mathcal{H}_{\beta}\left(\mathbb{R}^{3}\right) \cap C^{\infty}(V)$ with $\beta=2 / 3+6 / 5+m_{0}$, and, thanks to Theorem 6.5](iii), $F \in C^{1}\left(\mathbb{R}^{3}\right)$. Then $F \in \mathcal{X}(V)$ and, according to Theorem $5.3(\mathrm{i})$, we can apply Theorem 4.3 to obtain an infinite series asymptotic expansion for any non-trivial, decaying solution $y(t)$ of (1.4).

Example 6.13 (by A. D. Bruno). Below is a specific case when a solution has a similar, but different, asymptotic expansion. The system

$$
\begin{aligned}
y_{1}^{\prime}+y_{1} & =0 \\
y_{2}^{\prime}+3 y_{2} & =\frac{3}{2} y_{1}^{2} y_{2}^{1 / 3}
\end{aligned}
$$

has a solution $\left(y_{1}, y_{2}\right)=\left(e^{-t}, t^{3 / 2} e^{-3 t}\right)$ which, thanks to the term $t^{3 / 2}$, does not have an expansion (2.1).

We can examine system (6.25) and see that it does not satisfy the conditions in Theorems 4.3, 5.1 and 5.3. Indeed, we always require that each positively homogeneous function $F_{k}$ in approximations (4.3), (4.4), (5.1) of $F$ is infinitely differentiable in some neighborhood of any eigenvector of the matrix $A$, see Assumption 4.1)(ii) and Definition 5.2. In the current example,

$$
A=\left(\begin{array}{ll}
1 & 0 \\
0 & 3
\end{array}\right) \text { and } F\left(x_{1}, x_{2}\right)=\left(0, \frac{3}{2} x_{1}^{2} x_{2}^{1 / 3}\right) .
$$

Clearly, $\xi=(1,0)$ is an eigenvector of $A$ but $F_{1}=F$, with degree $\beta_{1}=2+1 / 3$, is not a $C^{\infty}$-function in any neighborhood of $\xi$. Thus, our results (Theorems 4.3, 5.1] and 5.3) cannot be applied to system (6.25).

Remark 6.14. In case $F$ is analytic, Lyapunov's First Method yields that a decaying solution solution $y(t)$ of (1.4) equals a series $\sum_{n=1}^{\infty} q_{n}(t) e^{-\mu_{n} t}$ for sufficiently large $t$, where $q_{n}(t)$ 's are some polynomials. See e.g. [1, Chapter I, §4] where the proof is based on the PoincaréDulac normal form. Bruno investigates a much larger class of equations of differential sums, which are not necessarily of a dissipative type like ours. He develops the theory of power geometry and finds solutions that have certain forms of asymptotic expansions. Specific algorithms are developed to calculate those asymptotic expansions. See [2 7] and references there in. His equations can have complex values, and the nonlinearity is comprised of power functions. His method and results are totally different from ours. For example, he does not obtain the particular expansion (1.3). Also, we obtain the asymptotic expansions for any given non-trivial, decaying solutions, and our nonlinearity, in case of real-valued functions, can contain more general terms such as in (2.6) and (6.21). 


\section{REFERENCES}

[1] Bibikov, Y. N. Local theory of nonlinear analytic ordinary differential equations, vol. 702 of Lecture Notes in Mathematics. Springer-Verlag, Berlin-New York, 1979.

[2] Bruno, A. D. Local methods in nonlinear differential equations. Springer Series in Soviet Mathematics. Springer-Verlag, Berlin, 1989.

[3] Bruno, A. D. Power geometry in algebraic and differential equations, vol. 57 of North-Holland Mathematical Library. North-Holland Publishing Co., Amsterdam, 2000.

[4] Bruno, A. D. Asymptotic behavior and expansions of solutions of an ordinary differential equation. Uspekhi Mat. Nauk 59, 3(357) (2004), 31-80.

[5] Bruno, A. D. Power-logarithmic expansions of solutions of a system of ordinary differential equations. Dokl. Akad. Nauk 419, 3 (2008), 298-302.

[6] Bruno, A. D. Power-exponential expansions of solutions of an ordinary differential equation. Dokl. Akad. Nauk 444, 2 (2012), 137-142.

[7] Bruno, A. D. On complicated expansions of solutions to ODES. Comput. Math. Math. Phys. 58, 3 (2018), 328-347.

[8] CaO, D., And Hoang, L. Asymptotic expansions in a general system of decaying functions for solutions of the Navier-Stokes equations. Ann. Mat. Pura Appl. (4) 199, 3 (2020), 1023-1072.

[9] CaO, D., And Hoang, L. Asymptotic expansions with exponential, power, and logarithmic functions for non-autonomous nonlinear differential equations. J. Evol. Equ. (2020), 1-47. In press, DOI:10.1007/s00028-020-00622-w.

[10] Cao, D., And Hoang, L. Long-time asymptotic expansions for Navier-Stokes equations with powerdecaying forces. Proc. Roy. Soc. Edinburgh Sect. A 150, 2 (2020), 569-606.

[11] Coddington, E. A., And Levinson, N. Theory of ordinary differential equations. McGraw-Hill Book Company, Inc., New York-Toronto-London, 1955.

[12] Cohen, P. J., And Lees, M. Asymptotic decay of solutions of differential inequalities. Pacific J. Math. 11 (1961), 1235-1249.

[13] Foias, C., Hoang, L., and Nicolaenko, B. On the helicity in 3D-periodic Navier-Stokes equations. I. The non-statistical case. Proc. Lond. Math. Soc. (3) 94, 1 (2007), 53-90.

[14] Foias, C., Hoang, L., and Nicolaenko, B. On the helicity in 3D-periodic Navier-Stokes equations. II. The statistical case. Comm. Math. Phys. 290, 2 (2009), 679-717.

[15] Foias, C., Hoang, L., Olson, E., and Ziane, M. On the solutions to the normal form of the Navier-Stokes equations. Indiana Univ. Math. J. 55, 2 (2006), 631-686.

[16] Foias, C., Hoang, L., Olson, E., And Ziane, M. The normal form of the Navier-Stokes equations in suitable normed spaces. Ann. Inst. H. Poincaré Anal. Non Linéaire 26, 5 (2009), 1635-1673.

[17] Foias, C., Hoang, L., And Saut, J.-C. Asymptotic integration of Navier-Stokes equations with potential forces. II. An explicit Poincaré-Dulac normal form. J. Funct. Anal. 260, 10 (2011), 3007-3035.

[18] Foins, C., And Saut, J.-C. Asymptotic behavior, as $t \rightarrow+\infty$, of solutions of Navier-Stokes equations and nonlinear spectral manifolds. Indiana Univ. Math. J. 33, 3 (1984), 459-477.

[19] Foias, C., And Saut, J.-C. Linearization and normal form of the Navier-Stokes equations with potential forces. Ann. Inst. H. Poincaré Anal. Non Linéaire 4, 1 (1987), 1-47.

[20] Foias, C., And Saut, J.-C. Asymptotic integration of Navier-Stokes equations with potential forces. I. Indiana Univ. Math. J. 40, 1 (1991), 305-320.

[21] Ghidaglia, J.-M. Long time behaviour of solutions of abstract inequalities: applications to thermohydraulic and magnetohydrodynamic equations. J. Differential Equations 61, 2 (1986), 268-294.

[22] Ghidaglia, J.-M. Some backward uniqueness results. Nonlinear Anal. 10, 8 (1986), 777-790.

[23] Hoang, L. Asymptotic expansions for the Lagrangian trajectories from solutions of the Navier-Stokes equations. Comm. Math. Physics 383, 2 (2021), 981-995.

[24] Hoang, L. T., and Martinez, V. R. Asymptotic expansion for solutions of the Navier-Stokes equations with non-potential body forces. J. Math. Anal. Appl. 462, 1 (2018), 84-113.

[25] Hoang, L. T., And Titi, E. S. Asymptotic expansions in time for rotating incompressible viscous fluids. Ann. Inst. H. Poincaré Anal. Non Linéaire 38 (2021), 109-137.

[26] Minea, G. Investigation of the Foias-Saut normalization in the finite-dimensional case. J. Dynam. Differential Equations 10, 1 (1998), 189-207. 
[27] SHI, Y. A Foias-Saut type of expansion for dissipative wave equations. Comm. Partial Differential Equations 25, 11-12 (2000), 2287-2331.

${ }^{1}$ Department of Mathematics and Statistics, Minnesota State University, Mankato, Mankato, MN 56001, U. S. A.

Email address: dat.cao@mnsu.edu

${ }^{2}$ Department of Mathematics and Statistics, Texas Tech University, 1108 Memorial CirCle, LubBock, TX 79409-1042, U. S. A.

Email address: luan.hoang@ttu.edu

${ }^{3}$ Department of Mathematics, University of North Georgia, Gainesville Campus, 3820 Mundy Mill RD., OAKWood, GA 30566, U. S. A.

Email address: thinh.kieu@ung.edu 